\title{
B-Spline Finite Elements and their Efficiency in Solving Relativistic Mean Field Equations
}

\author{
W. Pöschl \\ Physics-Department of the Duke University, \\ Durham, NC-27708, USA
}

(December 14, 2017)

\begin{abstract}
A finite element method using B-splines is presented and compared with a conventional finite element method of Lagrangian type. The efficiency of both methods has been investigated at the example of a coupled non-linear system of Dirac eigenvalue equations and inhomogeneous Klein-Gordon equations which describe a nuclear system in the framework of relativistic mean field theory. Although, FEM has been applied with great success in nuclear RMF recently, a well known problem is the appearance of spurious solutions in the spectra of the Dirac equation. The question, whether B-splines lead to a reduction of spurious solutions is analyzed. Numerical expenses, precision and behavior of convergence are compared for both methods in view of their use in large scale computation on FEM grids with more dimensions. A B-spline version of the object oriented $\mathrm{C}++$ code for spherical nuclei has been used for this investigation.
\end{abstract}

\section{PROGRAM SUMMARY}

Title of program: bspFEM.cc

Catalogue number:

Program obtainable from:

Computer for which the program is designed and others on which it has been tested: any Unix work-station.

Operating system: Unix

Programming language used: $\mathrm{C}++$

No. of lines in combined program and test deck:

Keywords: B-splines, Finite Element Method, Lagrange type shape functions, relativistic mean-field theory, mean-field approximation, spherical nuclei, Dirac equations, Klein-Gordon equations, classes

Nature of physical problem

The ground-state of a spherical nucleus is described in the framework of relativistic mean field theory in coordinate space. The model describes a nucleus as a relativistic system of baryons and mesons. Nucleons interact in a relativistic covariant manner through the exchange of virtual mesons: the isoscalar scalar $\sigma$-meson, the isoscalar vector $\omega$-meson and the isovector vector $\rho$-meson. The model is based on the one boson exchange description of the nucleon-nucleon interaction.

Method of solution

An atomic nucleus is described by a coupled system of partial differential equations for the nucleons (Dirac equations), and differential equations for the meson and photon fields (Klein-Gordon equations). Two methods are compared which allow a simple, self-consistent solution based on finite element analysis. Using a formulation based on weighted residuals, the coupled system of Dirac and Klein-Gordon equations is transformed into a generalized algebraic eigenvalue problem, and 
systems of linear and nonlinear algebraic equations, respectively. Finite elements of arbitrary order are used on uniform radial mesh. B-splines are used as shape functions in the finite elements. The generalized eigenvalue problem is solved in narrow windows of the eigenparameter using a highly efficient bisection method for band matrices. A biconjugate gradient method is used for the solution of systems of linear and nonlinear algebraic equations.

Restrictions on the complexity of the problem

In the present version of the code we only consider nuclear systems with spherical symmetry.

\section{LONG WRITE-UP}

\section{INTRODUCTION}

Over the last decade, the relativistic mean field theory (RMF) has been applied with great success to the description of low energy properties of nuclei [2] and to the description of scattering at intermediate energies []. Therefore, RMF gains increasing recognition. Effective models have been suggested [3, [1] which are represented by Lagrangians containing both, nucleonic and mesonic fields with coupling constants that have been adjusted to the many body system of nuclear matter and to finite nuclei in the valley of $\beta$-stability 45 . Of course, such a procedure is completely phenomenological and in spirit very similar to the non-relativistic density dependent HF-models (DDHF) of Skyrme and Gogny 66.7]. Compared to DDHF theory, the relativistic models seem to have important advantages: (i) they start on a more fundamental level, treating mesonic degrees explicitly and allowing a natural extension for heavy-ion reactions with higher energies, (ii) they incorporate from the beginning important relativistic effects, such as the existence of two types of potentials (scalar and vector) and the resulting strong spin-orbit term, a new saturation mechanism by the relativistic quenching of the attractive scalar field and the existence of anti-particle solutions, (iii) finally they are in many respects easier to handle than non-relativistic DDHF calculations.

Since the discovery of the halo phenomenon in light drip-line nuclei 8 the study of the structure of exotic nuclei has become a very exciting topic. Experiments with radioactive beams provide a lot of new data over entirely new ("exotic") regions of the chart of nuclides. On the theoretical side, presently existing models of the nucleus, relativistic ones as well as non-relativistic ones, have to be tested in these new regions in comparison with experiment. Improvements and extensions of the models become necessary.

Recent investigations 9, 10] have shown that coupling to the particle continuum and large extensions in coordinate space have to be taken into account in order to describe phenomena of exotic nuclear structure. The underlying equations of all nuclear models have therefore to be solved on discretizations in coordinate space. In contrast to "conventional" methods, based on expansions of the solution in basis functions with spherical or axial symmetry, sophisticated techniques have to be applied in order to solve the mean field equations in coordinate space.

With the non-relativistic HF-models extensive nuclear structure calculations have been performed based on the imaginary time method [11]. This very efficient method, however, is restricted to the non-relativistic cases where the single particle spectrum is limited from below. In relativistic model calculations, the imaginary time method would not converge due to mixing with negative energy states. Therefore, we plan a different approach with Krylov-subspace based methods 12 (for solutions on 2D and 3D meshes in coordinate space) and with the bisection method (1D spherical case [14]). In contrast to the imaginary time method, the required single particle or quasi particle eigenstates have to be calculated in each step of a self-consistent iteration. At first sight, it seems, that this approach is intractable since coordinate space discretizations on $2 \mathrm{D}$ or $3 \mathrm{D}$ finite element meshes lead to eigenvalue problems of large dimensions. With the block Lanczos method however, the calculation of eigenvalues and corresponding eigenvectors can be restricted to a small number which is required in the region of bound nucleons. In combination with the selfconsistent iteration method which is applied to the whole problem, the number of internal block Lanczos iterations can be reduced to corrections of the vectors which come from the previous iterations step of the selfconsistent loop. In references [13, 14] the solution of the spherical RMF equations and the spherical RHB equations with the finite element method in coordinate space has been demonstrated. In these investigations, I have observed that spurious solutions appear in the spectrum of eigenvalues of the Dirac operator of the RHB equations when they are discretized with finite elements of the Lagrangian type. Since the numerical cost to calculate eigensolutions on 1D-meshes is relatively small, it was not important to avoid spurious solutions a priori and therefore they have been eliminated by comparison 
of the number of nodes. In the $2 \mathrm{D}$ and $3 \mathrm{D}$ cases, however, it is important to reduce the size of the stiffness matrices to a minimum. This can be achieved by using shape functions with extremely good properties of interpolation, allowing wider meshes in coordinate space. Since B-splines are smooth, one would expect that they have the desired properties.

The major goal of the present paper is to give an answer to the question whether B-splines can improve the numerics in comparison to the often used shape functions of Lagrangian type. At the present state, our study is restricted to the solution of relativistic mean field equations. The results of our investigation are important with respect to large scale computations on finite element meshes of two and three dimensions. Such calculations are required in the relativistic mean field description of deformed exotic nuclei at low energies. I have worked out a B-spline version of the computer code which is published in 14] and compare the results obtained with both codes for spherical nuclei.

\section{THE RELATIVISTIC MEAN FIELD EQUATIONS}

The relativistic mean field model describes the nucleus as a system of nucleons which interact through the exchange of virtual mesons: the isoscalar scalar $\sigma$-meson, the isoscalar vector $\omega$-meson and the isovector vector $\rho$-meson. The model is based on the one boson exchange description of the nucleon-nucleon interaction. The effective Lagrangian density is 3

$$
\begin{aligned}
\mathcal{L}= & \bar{\psi}(i \gamma \cdot \partial-m) \psi \\
& +\frac{1}{2}(\partial \sigma)^{2}-U(\sigma)-\frac{1}{4} \Omega_{\mu \nu} \Omega^{\mu \nu}+\frac{1}{2} m_{\omega}^{2} \omega^{2}-\frac{1}{4} \overrightarrow{\mathrm{R}}_{\mu \nu} \overrightarrow{\mathrm{R}}^{\mu \nu}+\frac{1}{2} m_{\rho}^{2} \vec{\rho}^{2}-\frac{1}{4} \mathrm{~F}_{\mu \nu} \mathrm{F}^{\mu \nu} \\
& -g_{\sigma} \bar{\psi} \sigma \psi-g_{\omega} \bar{\psi} \gamma \cdot \omega \psi-g_{\rho} \bar{\psi} \gamma \cdot \vec{\rho} \vec{\tau} \psi-e \bar{\psi} \gamma \cdot A \frac{\left(1-\tau_{3}\right)}{2} \psi .
\end{aligned}
$$

Vectors in isospin space are denoted by arrows. The Dirac spinor $\psi$ denotes the nucleon with mass $m$. $m_{\sigma}, m_{\omega}$, and $m_{\rho}$ are the masses of the $\sigma$-meson, the $\omega$-meson, and the $\rho$-meson. $g_{\sigma}, g_{\omega}$, and $g_{\rho}$ are the corresponding coupling constants for the mesons to the nucleon. $e^{2} / 4 \pi=1 / 137.036$.

Since the relativistic mean field model has been described in a large number of articles, I omit a long discussion of the above given Lagrangian and the derivation of the RMF equations. Instead, I refer to section 2 of reference [13] and to section 2 of reference 114 . In these references, the development preceding to the investigations of the present paper is described in details. The main interest of the work presented below is focused on numerical aspects and performance of two FEM techniques in the solution of the RMF equations for spherical nuclei. In the following, I briefly list the static RMF equations for the spherical symmetric case.

Introducing spherical polar coordinates $(r, \theta, \phi)$, the Dirac equation reduces to a set of two coupled ordinary differential equations for the amplitudes $g(r)$ and $f(r)$ for proton and neutron states

$$
\begin{aligned}
& \left(\partial_{r}+\frac{\kappa+1}{r}\right) g(r)+(m+S(r)-V(r)) f(r)=-\varepsilon f_{i}(r), \\
& \left(\partial_{r}-\frac{\kappa-1}{r}\right) f(r)+(m+S(r)+V(r)) g(r)=+\varepsilon g(r),
\end{aligned}
$$

where the quantum number $\kappa= \pm 1, \pm 2, \pm 3, \ldots$. The scalar potential $S(r)$ and the vector potential $V(r)$ are composed of boson field amplitudes and coupling constants where

$$
S(r)=g_{\sigma} \sigma(r)
$$

and

$$
V(r)=g_{\omega} \omega^{0}(r)+g_{\rho} \tau_{3} \rho_{3}^{0}(r)+e \frac{\left(1-\tau_{3}\right)}{2} A^{0}(r)
$$

The symbols $g_{\sigma}, g_{\omega}, g_{\rho}$, and $e$ denote the coupling constants of the $\sigma$-field, the $\omega$-field, the $\rho$-field and the $A$-field, coupled to the nucleons. The meson fields $\sigma(r), \omega^{0}(r), \rho_{3}^{0}(r)$ and the photon field $A^{0}(r)$ are solutions of the inhomogeneous Klein-Gordon equations

$$
\begin{aligned}
& \left(-\partial_{r}^{2}-\frac{2}{r} \partial_{r}+\frac{l(l+1)}{r^{2}}+m_{\sigma}^{2}\right) \sigma(r)=-g_{\sigma} \rho_{\mathrm{s}}(r)-g_{2} \sigma^{2}(r)-g_{3} \sigma^{3}(r) \\
& \left(-\partial_{r}^{2}-\frac{2}{r} \partial_{r}+\frac{l(l+1)}{r^{2}}+m_{\omega}^{2}\right) \omega^{0}(r)=g_{\omega} \rho_{\mathrm{v}}(r)
\end{aligned}
$$




$$
\begin{aligned}
\left(-\partial_{r}^{2}-\frac{2}{r} \partial_{r}+\frac{l(l+1)}{r^{2}}+m_{\rho}^{2}\right) \rho^{0}(r) & =g_{\rho} \rho_{3}(r) \\
\left(-\partial_{r}^{2}-\frac{2}{r} \partial_{r}+\frac{l(l+1)}{r^{2}}\right) A^{0}(r) & =e \rho_{\mathrm{em}}(r)
\end{aligned}
$$

where the sources of the fields are the scalar density $\rho_{s}(r)$, the isoscalar baryon density $\rho_{v}(r)$, the isovector baryon density $\rho_{3}(r)$ and the electromagnetic charge density. They are composed of the nucleon wave functions in a bilinear way as

$$
\begin{aligned}
\rho_{\mathrm{s}}(r) & =\sum_{\kappa, n} n_{\kappa, n} \frac{(2|\kappa|}{4 \pi}\left(g_{\kappa, n}(r)^{2}-f_{\kappa, n}(r)^{2}\right) \\
\rho_{\mathrm{v}} & =\sum_{\kappa, n} n_{\kappa, n} \frac{2|\kappa|}{4 \pi}\left(g_{\kappa, n}(r)^{2}+f_{\kappa, n}(r)^{2}\right) \\
\rho_{3} & =\sum_{\kappa, n} n_{\kappa, n} \tau_{3 n} \frac{2|\kappa|}{4 \pi}\left(g_{\kappa, n}(r)^{2}+\mid f_{\kappa, n}(r)^{2}\right) \\
\rho_{\mathrm{em}} & =\sum_{\kappa, n} n_{\kappa, n} \frac{\left(1-\tau_{3 n}\right)}{2} \frac{2|\kappa|}{4 \pi}\left(g_{\kappa, n}(r)^{2}+f_{\kappa, n}(r)^{2}\right)
\end{aligned}
$$

where the quantities $n_{\kappa, n}$ are occupation numbers of the energy levels (indices $\kappa, n$ ). For the simple Hartree model without pairing, $n_{\kappa, n}=1$ for occupied levels and $n_{\kappa, n}=0$ for unoccupied levels. The index $n$ denotes the principal quantum number $(n=0,1,2, \ldots)$ and counts the eigensolutions of equation (2) from small to large energies $\varepsilon_{\kappa, n}$. The nucleon numbers filling an orbital $(\kappa, n)$ are taken into account by the factors $2|\kappa|$ in Eqs. (9) - (12). Since the densities (9) - (12) do not depend on the angular coordinates $\theta$ and $\phi$, no terms higher than of monopole order show up at the r.h.s. of Eqs. (8) - (8). Consequently, the solution of the these equations has to be restricted to $l=0$ in the description of spherical nuclei. For physical reasons, the nonlinear self-coupling of the $\sigma$-field has to be taken into account. It is described by the two terms $-g_{2} \sigma^{2}(r)$ and $-g_{3} \sigma^{3}(r)$ at the r.h.s. of Eq. (5). Without these terms, the RMF-model could not explain the compressibilities in finite nuclei and nuclear matter as well as the surface properties of finite nuclei.

\section{B-SPLINE AND LANGRANGIAN TYPE FINITE ELEMENTS}

The most widely used finite element type in many applications is the Lagrange type element. Lagrangian shape functions allow the simplest representation compared to other types of shape functions. For any finite element order $n$ they have the following expression in reference element representation (see Figs. 2a, 2c, 2e)

$$
N_{q}^{n}(\rho)=\prod_{\substack{l=0 \\ l \neq q}}^{n} \frac{(n \rho-l)}{(q-l)}
$$

where the coordinate $\rho$ is restricted to the interval $[0,1]$. From Eq. (13) it becomes obvious that Lagrange type finite elements are easy to handle. Generally, for any conventional finite element type, the shape functions (nodal basis) have the property

$$
N_{q}^{n}\left(q^{\prime} / n\right)=\delta_{q q^{\prime}}
$$

in the one dimensional case and

$$
N_{q}^{n}\left(\vec{\rho}_{q^{\prime}}\right)=\delta_{q q^{\prime}}, \quad \vec{\rho}=\left(\rho_{1}, \ldots, \rho_{M}\right)^{T}
$$

in the M-dimensional case where $\vec{\rho}_{q^{\prime}}$ denotes a grid point of M-dimensional finite elements. These shape functions form a so called nodal basis. Shape functions with property (15) can be constructed for finite elements of any geometrical form as for example triangular elements or quadratic elements in two dimensions and tetraedric or cubic elements in three dimensions. Also the location of the 
mesh points which belong to a finite element can be distributed in almost arbitrary manner over the domain of the element. In most cases, however, M-cube elements (intervals, squares, cubes, etc.) with a uniform distribution of the nodes are sufficient and allow extremely efficient calculations of the stiffness matrices for a given boundary value problem. The shape functions of such elements are represented as products of Lagrange polynomials (13)

$$
N_{\left(q_{1}, \ldots, q_{M}\right)}^{\left(q_{1}, \ldots, q_{M}\right)}\left(\rho_{1}, \ldots, \rho_{M}\right)=\prod_{i=1}^{M} N_{q_{i}}^{n_{i}}\left(\rho_{i}\right), \quad q_{i}, \ldots, n_{i}
$$

where $n_{i}$ denotes the order of the element in the direction of dimension $i$ and $(q)=\left(q_{1}, \ldots, q_{M}\right)$ forms the index tuple of the nodes.

The construction (16) of Lagrange type M-cube shape functions shows that 1-dimensional Lagrange type finite elements allow the most simple generalization to M-cube meshes. A great advantage of such shape functions can also be seen from a technical view point. Implementations of the general M-dimensional case in object oriented programming styles become simple. The amount of data required by an object which represents a Lagrangian M-cube finite element as reference element is almost the same as in the 1-dimensional case if the orders $n_{1}, \ldots, n_{M}$ are equal. In this special case the data representing all shape functions of a M-cube element comprise $2 \cdot n_{i} \cdot n_{i}^{G}$ floating point numbers where I denote by $n_{i}^{G}$ the number of Gauss points on a Gaussian mesh in dimension $i$. In the more general case where $n_{i} \neq n_{j}$ for $i \neq j$ and $n_{i}^{G} \neq n_{j}^{G}$ for $i \neq j$ the amount of float point numbers required to represent all shape functions is

$$
2 \sum_{i=1}^{M} n_{i} n_{i}^{G}
$$

which is still small compared to the number of values

$$
2 \prod_{i=1}^{M} n_{i} n_{i}^{G}
$$

required for shape functions of arbitrary type and arbitrary distribution of the nodes over the element.

The numerical cost for the integration of matrix elements reduces dramatically in cases where operators split up into products of operators each depending on a complementary subset of the coordinates $\rho_{1}, \ldots, \rho_{M}$. In the most ideal case an operator factorizes completely leading with Eq. (16) to a complete factorization of matrix elements.

$$
\left\langle N_{\left(q_{1}, \ldots, q_{M}\right)}^{\left(n_{1}, \ldots, n_{M}\right)}\left(\rho_{1}, \ldots, \rho_{M}\right)\left|\prod_{i=1}^{M} \hat{O}_{i}\left(\rho_{i}\right)\right| N_{\left(q_{1}^{\prime}, \ldots, q_{M}^{\prime}\right)}^{\left(n_{1}, \ldots, n_{M}\right)}\left(\rho_{1}, \ldots, \rho_{M}\right)\right\rangle=\prod_{i=1}^{M}\left\langle N_{q_{i}}^{n_{i}}\left(\rho_{i}\right)\left|\hat{O}_{i}\left(\rho_{i}\right)\right| N_{q_{i}^{\prime}}^{n_{i}}\left(\rho_{i}\right)\right\rangle .
$$

This becomes obvious when I rewrite Eq. (17) in terms of a numerical Gauss integration

$$
\sum_{l_{1}, \ldots, l_{M}}^{N} N_{(q)}^{(n)}\left(\rho_{1}^{l_{1}}, \ldots, \rho_{M}^{l_{M}}\right) \hat{O}\left(\rho_{1}^{l_{1}}, \ldots, \rho_{M}^{l_{M}}\right) N_{(q)}^{(n)}\left(\rho_{1}^{l_{1}}, \ldots, \rho_{M}^{l_{M}}\right) \prod_{i=1}^{M} w_{l_{i}}=\prod_{i=1}^{M} \sum_{l_{i}=1}^{N} N_{q_{i}}^{n_{i}}\left(\rho_{i}^{l_{i}}\right) \hat{O}_{i}\left(\rho_{i}^{l_{i}}\right) N_{q_{i}^{\prime}}^{n_{i}}\left(\rho_{i}^{l_{i}}\right)
$$

where, assuming that $\mathrm{N}$ is the number of Gauss points in each coordinate, the number of floating point operations on the left hand side is greater than $4 N^{M}$ while on the r.h.s. it is smaller than $3 \mathrm{MN}$. 


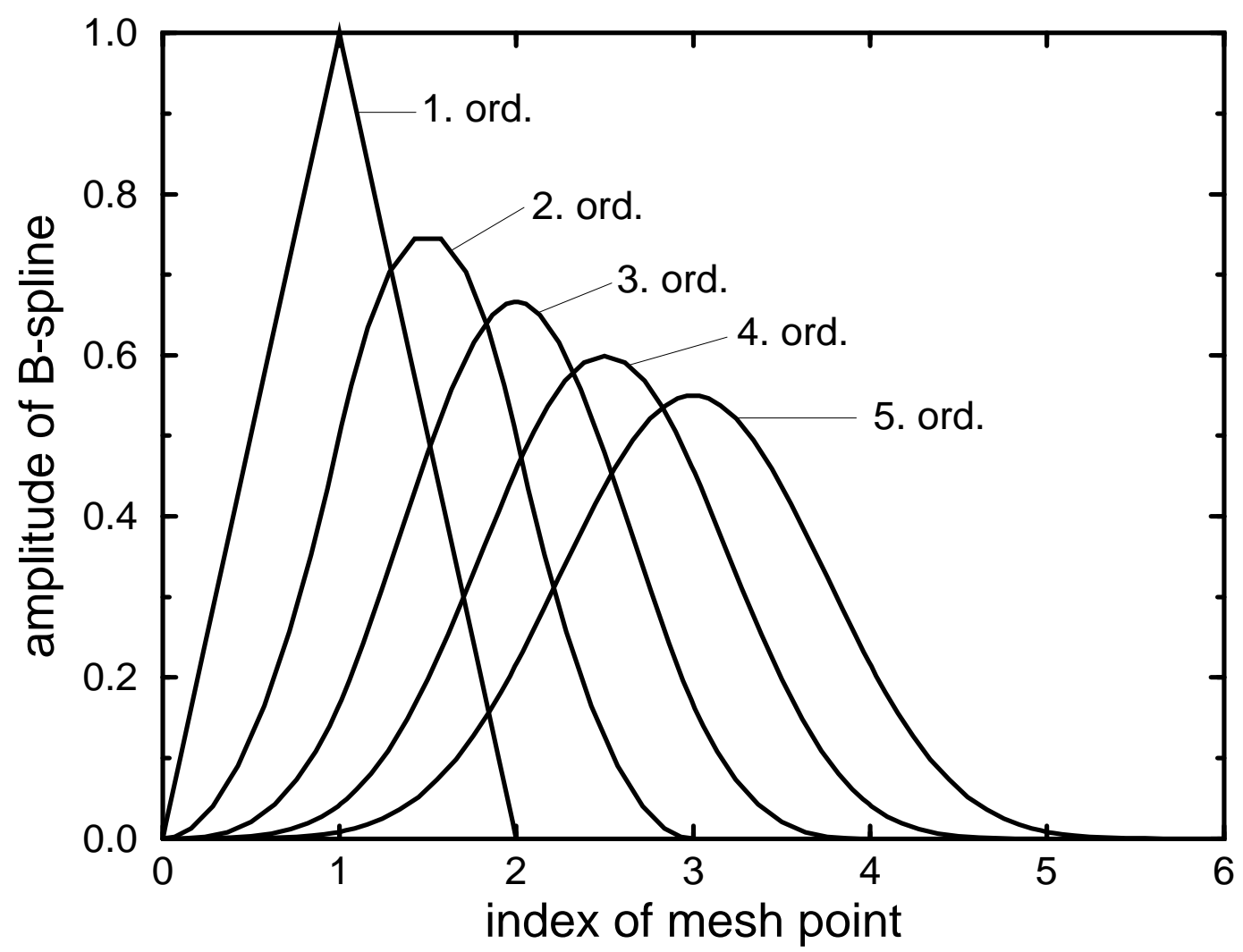

Fig. 1: B-spline functions of different orders increasing from 1 to 5 .

These advantages of Lagrangian type finite elements gave us a reason to apply them in several previous studies and calculations (see references 13 15, 96). In these references, it has been shown that Lagrangian finite elements provide an excellent tool for solving the equations of the RMF model in self-consistent iterations in coordinate space. In this manuscript, I present a new finite element technique using B-splines as shape functions and compare this method with the FEM based on Lagrangian shape functions. B-splines have a compact support and are defined as polynomials piecewise on intervals which are bounded by neighbored mesh points. The basic criterium in the construction of these basis functions is optimized smoothness over the whole support. This property is guaranteed if all derivatives up to the order $n-1$ obey the conditions of continuity in all matching points of the mesh. The order $n$ of a given B-spline corresponds to the degree of the polynomials by which it is composed. In Fig. 1, examples are shown for B-splines of order one to five. $n+2$ mesh points are required to construct a B-spline of the order $n$. In contrast to Lagrangian shape functions, B-splines of any order do not change sign. A common property of both types of shape functions is expressed by

$$
\sum_{p} N_{p}(\rho)=\sum_{p} B_{p}(\rho)=1
$$

where $p$ denotes the mesh point index. The fact, that B-splines of any order satisfy all these conditions makes it impossible to find an expression in closed form in the sense of Eq. (13). Rather, they are generated by the following brief algorithm.

$$
\begin{aligned}
\text { start: } & B_{i, 1}(x)=\left\{\begin{array}{ll}
\left(\left(x_{i+1}-x_{i}\right)\right)^{-1} & x_{i} \leq x \leq x_{i+1} \\
0 & x<x_{i}, x>x_{i+1}
\end{array} \quad i=0, \ldots, n\right. \\
B_{i, k}(x) & =\frac{\left(x-x_{i}\right)}{\left(x_{i+k}-x_{i}\right)} B_{i, k-1}(x)+\frac{\left(x_{i+k}-x\right)}{\left(x_{i+k}-x_{i}\right)} B_{i+1, k-1}(x)
\end{aligned}
$$


I define a B-spline finite element as a region which is bounded by two neighboring mesh points in the one-dimensional case or as a M-cube where the $2^{M}$ corners are identical with the $2^{M}$ mesh points of a cubic grid which are closest to the center of the cube. Obviously, this definition is restricted to cubic grids but it will turn out to be extremely efficient in all cases where cubic finite element discretizations can be applied.

The figures $2 \mathrm{~b}, 2 \mathrm{~d}$, and $2 \mathrm{f}$ display $3^{\text {rd }}$ order, $4^{\text {th }}$ order and $5^{\text {th }}$ order B-spline finite elements in one dimension according to our definition. The figures show, that all parts of a B-spline are contained in each element.

Fig. $2 a$

Fig. $2 b$
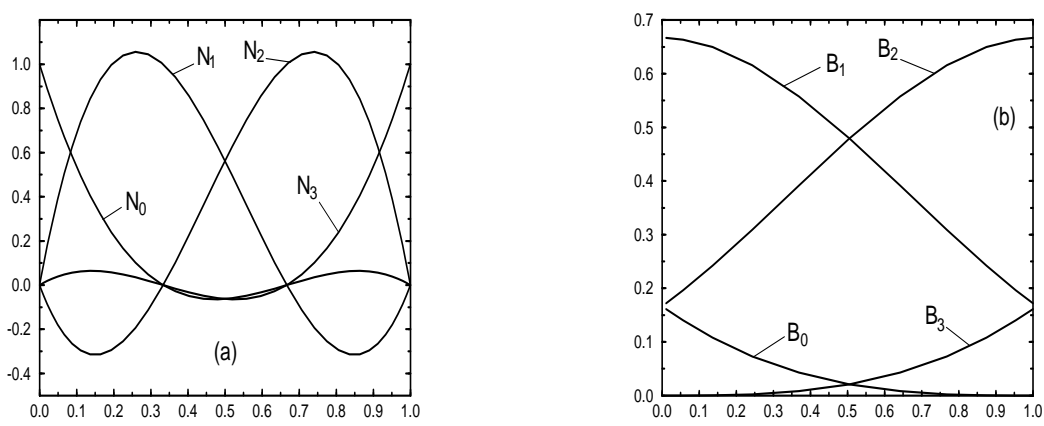

Fig. $2 c$

Fig. $2 d$
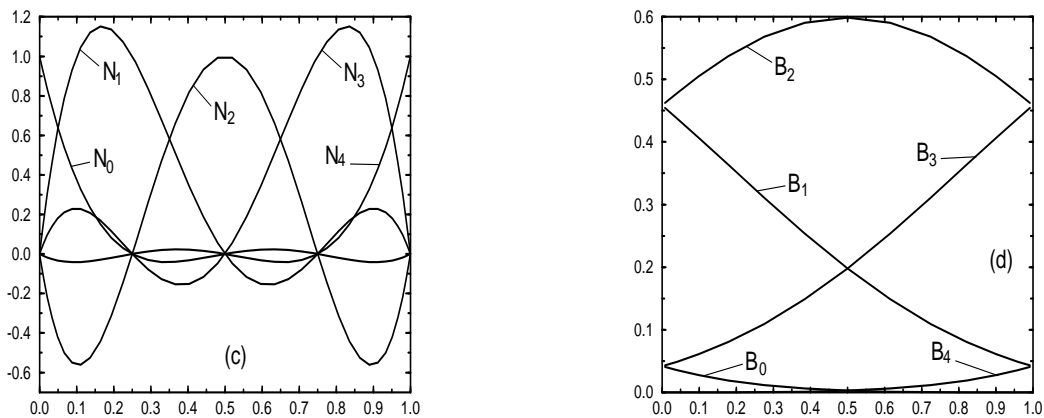

Fig. $2 e$
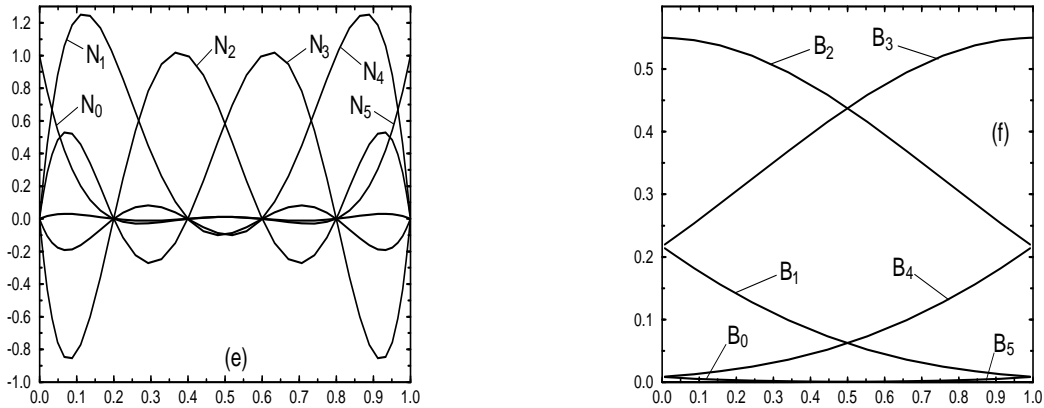

Fig. 2: $\quad$ Finite elements in reference element representation. In the figures (a),(c),(e), Lagrange type elements with corresponding shape functions are shown for $3^{\text {rd }}$ order, $4^{\text {th }}$ order and $5^{\text {th }}$ order. In comparison, B-spline elements of $3^{\text {rd }}$ order, $4^{\text {th }}$ order and $5^{\text {th }}$ order are displayed in the figures (b), (d), (f). 
A B-spline of order $n$ is determined by $n+2$ mesh-points through the algorithm (22) and the support is composed by $n+1$ intervals. However, the pieces which are contained in a single element belong to different B-splines each located at another mesh point and determined by a different set of mesh points. They are the overlap sections of all splines which are not zero in the considered element. This fact has to be taken into account especially if nonuniform meshes are used. As a consequence, the amplitudes of the shape functions in such a finite element depend on the position of all mesh points which are in the support of all contributing B-splines. Most of these mesh points are outside of the element and belong to other elements. Therefore, the degree of "interaction" between neighbored elements is maximized and much higher than for elements of Lagrange type where only next neighbor elements "interact" through their outermost contact grid points. This leads to a strong reduction of the total number of required mesh points as I will demonstrate in section 5 .

A disadvantage, however, appears when non-uniform meshes are used. As it has been discussed in reference [14, Lagrangian elements can be mapped on a reference element through linear affine transformations. This is even possible for non-uniform meshes. Therefore, Lagrangian shape functions need to be evaluated only once in the reference element and amplitudes and abscissas can be accessed by means of a pointer to that reference element. These advantages are also valid for B-splines as long as uniform meshes are used. In the case of non-uniform meshes the algorithm (22) has to be evaluated for each argument taken on the global region. This leads to a reduction of storage requirement but increases the numerical cost by a factor which corresponds to the number of floating point operations which are necessary to carry out the scheme (22). Consequently, the numerical effort in the calculation of the stiffness matrices of a given problem increases by the same factor. On the other hand, the number of algebraic equations resulting from a finite element discretization is usually large and the numerical cost to solve these equations increases faster with the number of equations ( $\sim$ number of mesh points) than the numerical cost in the calculation of the stiffness matrices with the number of mesh points. This trend is even enhanced for increasing dimension $M$ of the descretization where the condition of the stiffness matrices becomes worse. B-splines finite elements might therefore be superior in multidimensional FEM discretizations as compared to Lagrangian finite elements.

\section{THE FEM DISCRETIZATION}

A basic principle of FEM is the approximation of the solution for a given problem in a space of shape functions which have compact support and existing continuous weak derivative of maximum degree $\mathrm{m}$. Together with a p-norm which is for all those functions defined as

$$
\|\varphi\|_{m, p}:=\left(\sum_{\alpha=0}^{m} \int_{\Omega}\left|D^{\alpha} \varphi(x)\right|^{p}\right)^{1 / p}
$$

the above given space is a Banach space. According to the norm it is usually called Sobolev space $W_{p}^{m}(\Omega)$ where $\Omega$ may be any compact domain of the coordinate space. Since $W_{p}^{m}(\Omega)$ is complete, the solutions of any partial differential equation of an order not higher than $\mathrm{m}$ can be approximated to arbitrary precision in $W_{p}^{m}(\Omega)$ on the whole domain $\Omega$. This property plays an important role for the solution of differential equations with computational methods because finite element discretizations of the domain $\Omega$ correspond to subsets of linear independent functions of $W_{p}^{m}(\Omega)$ and because the representation of functions of $W_{p}^{m}(\Omega)$ on the computer is simple. In FEM, $\Omega$ is subdivided into a large number of small sub-domains which are called finite elements. Each element is support of a certain number of shape functions which is equivalent to the number of constraints set on the element. These functions span up a finite element space. The corners of the elements are located on a finite element mesh. However, additional mesh points can exist in the interior or on the surface of each element and additional constraints as derivatives of any order can be applied. In such cases the order of the element is higher than first order.

In the following, I discuss the finite element discretization of the Eqs. (2) and Eqs (5) to (8) for both types Lagrangian and B-spline elements. In the present application a nodal basis $\left\{N_{p}(r)\right\}$ is used in the case of Lagrange elements and a non-nodal basis $\left\{B_{p}(r)\right\}$ is used in the case of B-spline elements. Examples for discretizations with elements of $3^{\text {rd }}$ order are displayed for both types in Fig. 3a and 3b. Each Lagrangian element in Fig. 3a has two boundary nodes and two additional nodes in the interior whereas the B-spline elements in Fig. $3 \mathrm{~b}$ are free of interior nodes. In the B-spline 
method additional nodes are required outside of the region of integration in order to generate the shape functions which have non-zero overlap with the inner region. I use the notation $N_{p}(\rho)$ and $B_{p}(\rho)(0 \leq q \leq n)$ for shape functions in reference element representation, and $N_{p}(r)$ or $B_{p}(r)$ $\left(1 \leq p \leq n^{\text {nod }}\right)$ for shape functions $N_{p}$ and $B_{p}$ on the global mesh in the r-coordinate space. Using the standard representation for the Pauli matrices, the Dirac equation (2) is written in matrix form

$$
\left[\left(\partial_{r}+r^{-1}\right) \cdot \sigma_{3} \sigma_{1}-\kappa r^{-1} \cdot \sigma_{1}+(m+S(r)) \cdot \sigma_{3}+V(r) \cdot \mathbf{1}_{2}\right] \Phi(r)=\varepsilon \mathbf{1}_{2} \Phi(r) .
$$

Fig. 3a
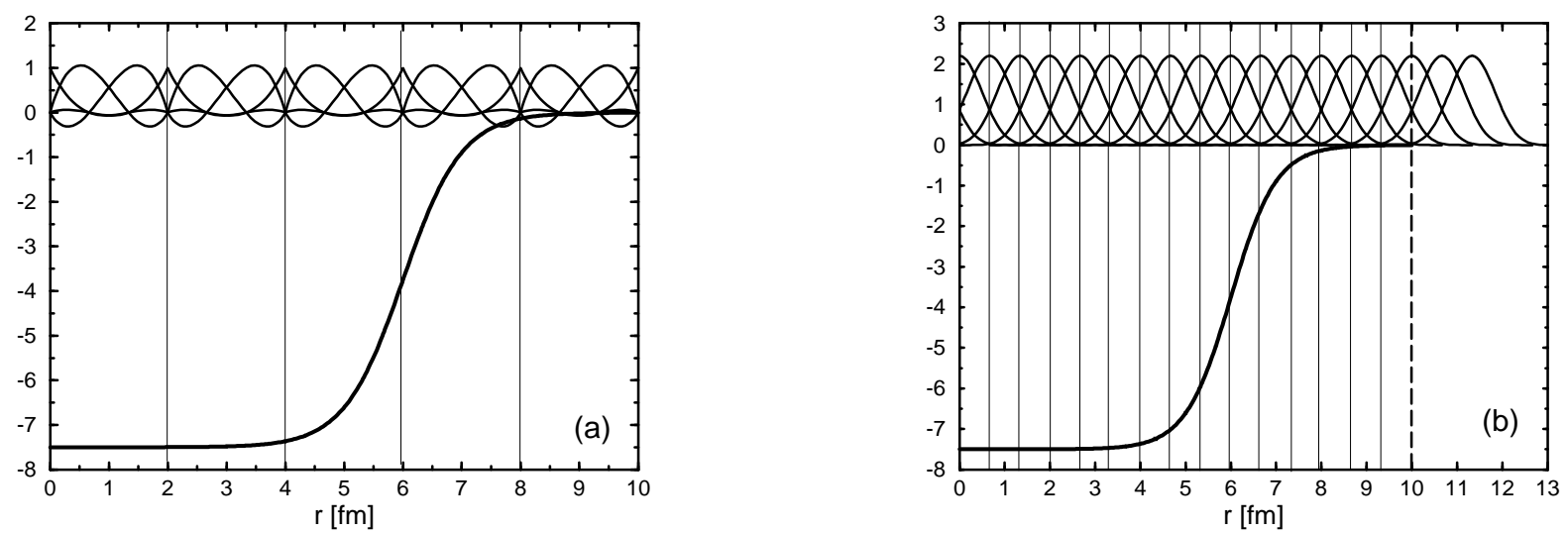

Fig. 3: Global discretizations with $3^{\text {rd }}$ order finite elements (a) of Lagrangian type and (b) of B-spline type. In both examples a total number of 16 mesh points is used in the region between $r=0 \mathrm{fm}$ and $r=10 \mathrm{fm}$.

For the nucleon spinor I use the FEM ansatz

$$
\Phi(r)=\sum_{p} u_{p} B_{p}(r)
$$

with B-spline functions $B_{p}(r)$ and node variables $u_{p}:=\left(u_{p}^{(g)}, u_{p}(f)\right)^{T}$. In a Lagrangian FEM ansatz, the shape functions $B_{p}(r)$ defined in (25) are replaced by the shape functions $N_{p}(r)$. In the weak formulation of the eigenvalue problem (2), the weighted residual (see [14) leads to algebraic equations of the form

$$
\begin{array}{r}
\sum_{p}\left\langle w_{p^{\prime}}(r)\left|\left[\left(\partial_{r}+r^{-1}\right) \cdot \sigma_{3} \sigma_{1}-\kappa r^{-1} \cdot \sigma_{1}+(m+S(r)) \cdot \sigma_{3}+V(r) \cdot \mathbf{1}_{2}\right]\right| B_{p}(r)\right\rangle u_{p}= \\
\varepsilon\left\langle w_{p^{\prime}}(r)\left|\mathbf{1}_{2}\right| B_{p}(r)\right\rangle u_{p}
\end{array}
$$

with weighting functions $w_{p^{\prime}}(r)$. The weighting functions are chosen

$$
w_{p^{\prime}}(r)=\left(1-\left(\frac{r}{r_{\max }}\right)^{2}\right) r^{2} r^{l_{g / f}} B_{p}(r)
$$

in the case of B-spline elements or

$$
w_{p^{\prime}}(r)=\left(1-\left(\frac{r}{r_{\max }}\right)^{2}\right) r^{2} r^{l_{g / f}} N_{p}(r)
$$

when Lagrangian shape functions are used in ansatz (25).

$$
l_{g}= \begin{cases}-\kappa-1 & \kappa<0 \\ \kappa & \kappa>0\end{cases}
$$




$$
l_{f}=\left\{\begin{array}{cc}
-\kappa & \kappa<0 \\
\kappa-1 & \kappa>0
\end{array}\right.
$$

The factor $r^{2}$ corresponds to the Jacobi determinant of the transformation into spherical coordinates and compensates singularities in the operator. The factor $r^{l_{g}}$ or $r^{l_{f}}$ respectively includes boundary conditions at $r=0$ properly for upper $(g(r))$ and lower components $(f(r))$ of the spinor $\Phi(r)$. The factor $\left(1-\left(r / r_{\max }\right)^{2}\right)$ includes boundary conditions $g\left(r_{\max }\right)=0$ at $r=r_{\max }$ in matrix elements which are multiplied with g-components in $\Phi(r)$ whereas this factor is replaced by 1 when a matrix element is multiplied with node variables of the $f(r)$-component.
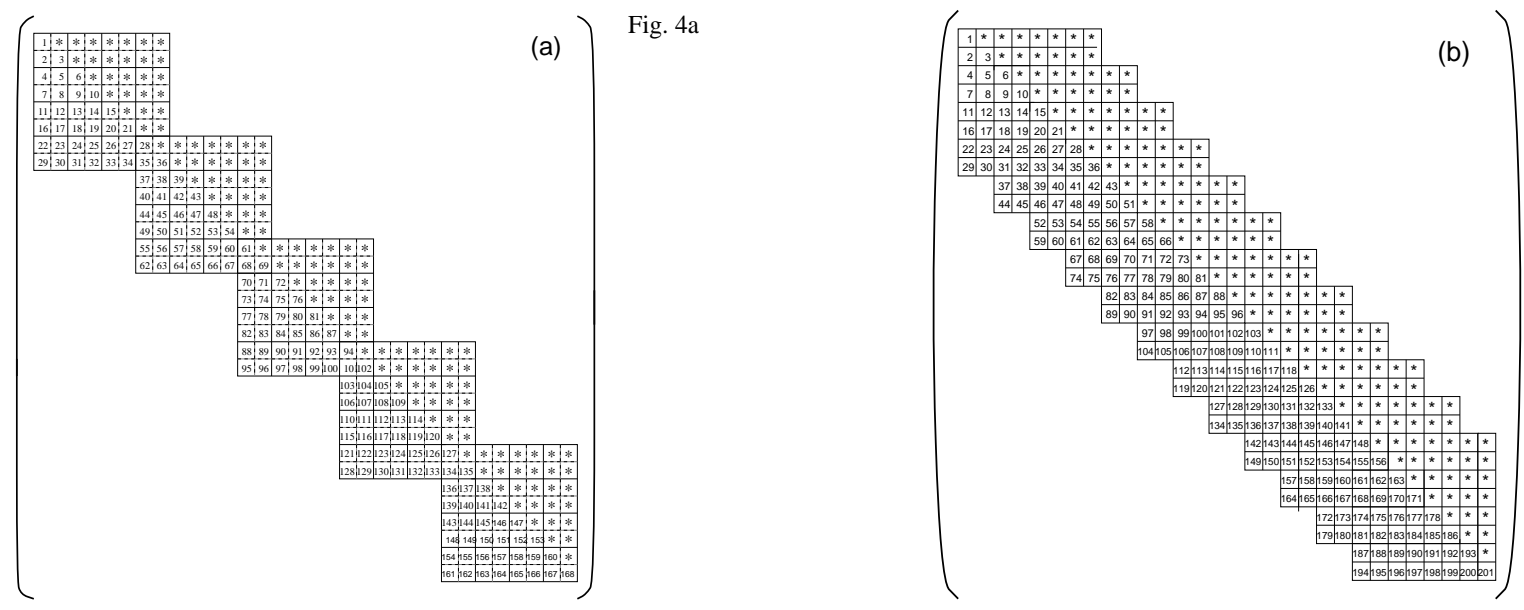

Fig. 4b

Fig. 4a
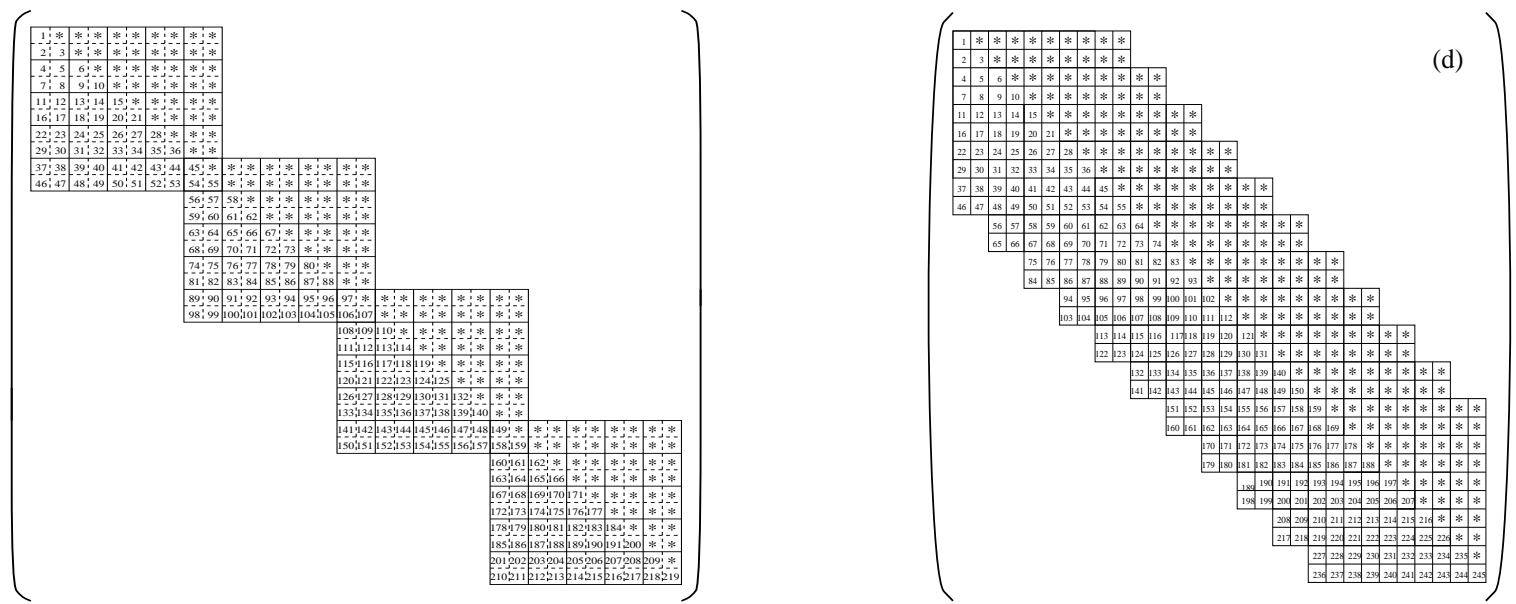

Fig. 4d 

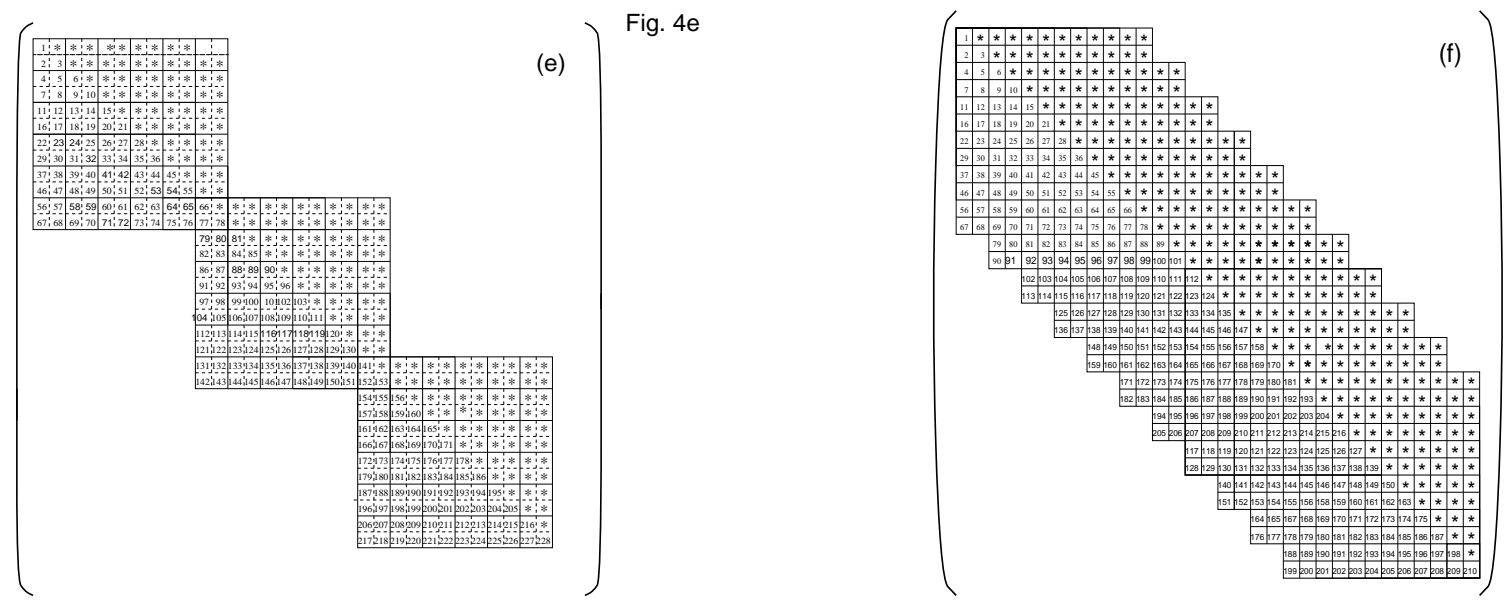

Fig. $4 f$

Fig. 4: Occupation patterns of stiffness matrices which result from the finite element discretization of Eq. (2). The figures (a), (c), (e) display matrices which correspond to $3^{\text {rd }}$ order, $4^{\text {th }}$ order and $5^{\text {th }}$ order Lagrange type FEM. Figues (b), (d), (f) display patterns which result from $3^{\text {rd }}$ order, $4^{\text {th }}$ order and $5^{\text {th }}$ order B-spline FEM discretizations. The numbers represent counter indices used in a vector storage technique.

The boundary conditions at $r=0 \mathrm{fm}$ depend on the quantum number $\kappa$ and are defined in the following way.

$$
\begin{aligned}
& f(r=0)=0 \quad \text { for } \quad \kappa=-1 \\
& g(r=0)=0 \quad \text { for } \quad \kappa=+1 \\
& g(r=0)=0 \quad \text { and } \quad f(r=0)=0 \text { for }|\kappa|>1 \text {. }
\end{aligned}
$$

The system of algebraic equations (26) forms a generalized eigenvalue problem of the form $A u=$ $\varepsilon N u$ with stiffness matrices $A$ and $N$ can be analyzed from the resulting matrix equation

$$
\left[S_{1} \otimes \sigma_{3} \sigma_{1}+S_{2} \otimes \sigma_{3} \sigma_{1}-\kappa S_{2} \otimes \sigma_{1}+m S_{3} \otimes \sigma_{3}+S_{4} \otimes \sigma_{3}+S_{5} \otimes \mathbf{1}_{2}\right] u=\varepsilon\left[S_{3} \otimes \mathbf{1}_{2}\right] u
$$

where $u$ is a vector with components $\left(u_{1}^{(g)}, u_{1}^{(f)}, \ldots, u_{n}^{(g)}, u_{n}^{(f)}\right)^{T}$. The symbols $S_{1}$ to $S_{5}$ denote the stiffness matrices of the operators on the l.h.s. of Eq. (24),

$$
\begin{aligned}
& S_{1}=\left\langle w_{p^{\prime}}(r)\left|\partial_{r}\right| B_{p}(r)\right\rangle, \\
& S_{2}=\left\langle w_{p^{\prime}}(r)\left|r^{-1}\right| B_{p}(r)\right\rangle, \\
& S_{3}=\left\langle w_{p^{\prime}}(r) \mid B_{p}(r)\right\rangle, \\
& S_{4}=\left\langle w_{p^{\prime}}(r)|S(r)| B_{p}(r)\right\rangle, \\
& S_{5}=\left\langle w_{p^{\prime}}(r)|V(r)| B_{p}(r)\right\rangle .
\end{aligned}
$$

In Figs. 4a-f, occupation patterns of the stiffness matrices A are displayed for Lagrangian and Bspline finite element discretizations. The matrices in Figs. 4a, 4c, 4e result from the Lagrange FEM with $3^{\text {rd }}$ order, $4^{\text {th }}$ order and $5^{\text {th }}$ order finite elements. For comparison, I show the corresponding stiffness matrices of the B-spline FEM in Figs. 4b, 4d, 4f. The sub-block structure of $2 \times 2$-blocks in all matrices results from the fact that Eq. (2) is a system of two coupled equations. The number of occupied $2 \times 2$-blocks for a given order $n^{\text {ord }}$ in the Lagrange FEM is $n^{f e} \cdot\left[\left(n^{\text {ord }}\right)^{2}+2 n\right]+1$ while in the B-spline method the occupation increases to $n^{f e} \cdot\left[2\left(n^{\text {ord }}\right)^{2}+n\right]+1 \cdot n^{f e}$ denotes here for both cases the number of finite elements used in the Lagrange method and is different from the number of elements which is used in the B-spline FEM. 
The FEM discretization of the Klein-Gordon equations (5) to (8) is described in the appendix. Finally, the coupled system of differential equations (2), (8) to (8) is replaced by a system of linear algebraic equations

$$
A\left(\vec{\sigma}, \vec{\omega}^{0}, \vec{\rho}^{0}, \vec{A}^{0}\right) u=\varepsilon N u
$$

for the node variables $u_{p}^{(g)}, u_{p}^{(f)}$ of nucleon spinors, and

$$
\begin{aligned}
B_{\sigma}\left(\vec{\sigma}_{\text {old }}\right) \vec{\sigma} & =\vec{r}^{(\mathrm{s})} \\
B_{\omega} \vec{\omega}^{0} & =\vec{r}^{(\mathrm{v})} \\
B_{\rho} \vec{\rho}^{0} & =\vec{r}^{(3)} \\
B_{A} \vec{A}^{0} & =\vec{r}^{(\mathrm{em})}
\end{aligned}
$$

for the node variables $\sigma_{p}, \omega_{p}, \rho_{p}$, and $A_{p}$ of the meson fields $\sigma(r), \omega^{0}(r), \rho^{0}(r)$, and photon field $A^{0}(r)$. The occupation patterns of the matrices $B_{\sigma}, B_{\omega}, B_{\rho}$, and $B_{A^{0}}$ for various shape functions are very similar to those of the matrix A (Figs. $4 \mathrm{a}$-d). The main difference is that $2 \times 2$-blocks have to be replaced by single matrix elements.

\section{ANALYSIS OF SPURIOUSITY}

The appearance of spurious solutions in applications of FEM is a well known problem in general. First applications of the finite element method in relativistic nuclear physics 13 have shown that spurious solutions appear in the spectra of the Dirac equation. Linear finite elements have been used to calculate solutions of the relativistic nuclear slab model. Comparisons of the solutions with solutions that have been obtained with other numerical techniques (shooting method) have shown that FEM reproduces the physical spectra very well and that spurious solutions have no influence. In a further step 14] Lagrangian finite elements of 1 . to $4^{\text {th }}$ order have been used in the self-consistent solution of the RMF equations of sperical nuclei. In these calculations, it has been shown (up to $4^{\text {th }}$ order) that the density of spurious solutions in the spectra decreases for increasing order of the elements.

In this sections, I present a systematic study of the spurious spectra which appear in the spherical symmetric case. In the initial step of a self-consistent ground state calculation of ${ }_{82}^{208} \mathrm{~Pb}$, WoodsSaxon potentials

$$
\begin{aligned}
& \left.S(r)=S(0)\left(1+\exp \left(\frac{r-r_{s}}{a}\right)\right)\right)^{-1}, \\
& V(r)=V(0)\left(1+\exp \left(\frac{r-r_{s}}{a}\right)\right)^{-1},
\end{aligned}
$$

are used for the scalar potential $S(r)$ and for the vector potential $V(r)$. For ${ }^{208} \mathrm{~Pb}$ the values of these potentials at $r=0 \mathrm{fm}$ are chosen $S(0)=-395 \mathrm{MeV}$ and $V(0)=320 \mathrm{MeV}$, respectively. $a=0.5 \mathrm{fm}$ and $r_{s}=9.0 \mathrm{fm}$. The calculation is performed on a uniform radial mesh extending from $r_{\min }=0 \mathrm{fm}$ to $r_{\max }=20 \mathrm{fm}$. A smaller value $r_{\max }=12 \mathrm{fm}$ would be sufficient for ${ }^{208} \mathrm{~Pb}$ to obtain good approximations of the bound single particle states. For a good resolution of the continuum, however, a large extension of the mesh in coordinate space is necessary. An extremely high number of 200 mesh points has been used in the calculation of the sprectra shown in Fig. 5a to Fig. 5f. The reason for that choice will become clear from the subsequent discussion of Fig. 6. For a nucleon mass of $939 \mathrm{MeV}$ (parameter set NL3), the Dirac gap extends from $-939 \mathrm{MeV}$ to $+939 \mathrm{MeV}$. Bound solutions are expected to have energies which are located in the Dirac gap. In the following calculations an energy window ranging from $-1300 \mathrm{MeV}$ to $+1300 \mathrm{MeV}$ has been chosen which covers parts of the lower and upper continuum as well.

The results which are presented in the subsequent discussion correspond to the first iteration step and a value $\kappa=-1$ (s-waves). Spurious spectra of similar eigenvalue distributions are obtained for all other $\kappa$-values $(\kappa=+1, \pm 2, \pm 3, \ldots)$. Disregarding the fact that the eigensolutions change while they converge, very similar results are found in all iteration steps of the selfconsistent iteration.

One of the most interesting questions to be answered in the present paper is, whether the appearance of spurious solutions can be avoided using B-spline finite elements instead of Lagrangian elements. Since both methods are identical in the case of $1^{\text {st }}$ order, spurious solutions appear also 
in the B-spline FEM. However, from that one can not conclude that spurious solutions appear in FEM discretizations with B-splines of higher order. The following six figures Fig. 5a to Fig. 5f display energy spectra of Eq. (2) which have been calculated for many different orders with both methods, the Lagrange FEM and the B-spline FEM. In Fig. 5a and Fig. 5b the positive and negative spectra are shown for $1^{\text {st }}$ order to $4^{\text {th }}$ order finite elements. The white circles correspond to physical eigenvalues which have been calculated with Lagrange type elements. They are located at the same energies as the white triangles which correspond to physical eigenvalues obtained with the B-spline FEM. The figures show that the physical spectra are independent on the order and on the used method. However, the number of black filled circles and filled triangles in the spectra decreases for increasing order of the used shape functions. The filled symbols indicate eigenvalues of spurious solutions. It turns out that the distributions of spurious eigenvalues over the entire energy range are identical for both methods and in all orders. In comparison to the Lagrange FEM, the B-spline method does obviously not reduce the number of spurious states as long as the order is the same used in both methods. However, for both methods, the density of spurious solutions in the spectra can be strongly reduced by increasing the order of the finite elements. Particularly, from Fig. 5c to Fig. 5f, on can see, that the spurious eigenvalues drift away from the Dirac gap when the order is increased. Consequently, for any energy window there exists an order which is high enough so that no spurious solutions appear in the window. An exception forms the region between the lowest positive physical eigenvalue and the highest negative physical eigenvalue. For all element orders with both methods, no spurious solution has been found in that region.

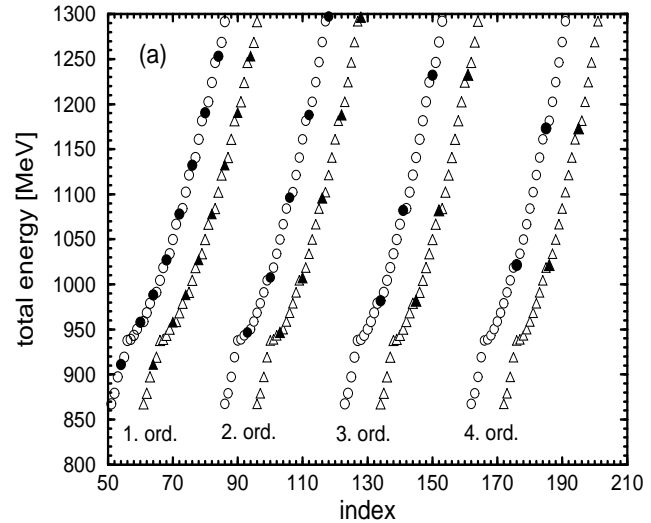

Fig. $5 \mathrm{c}$

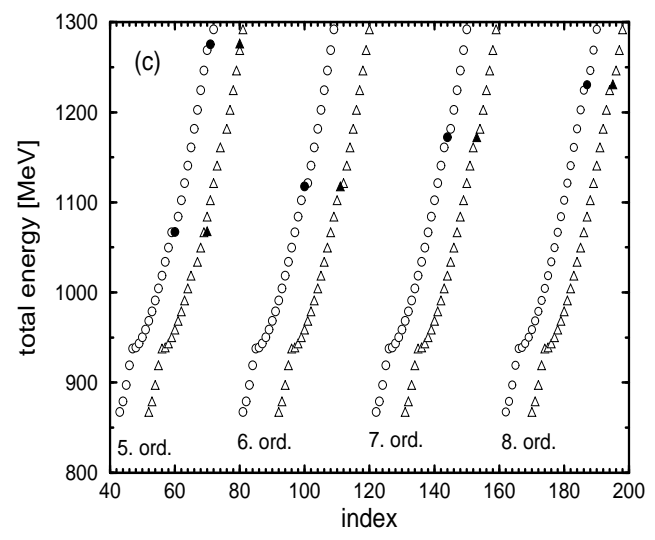

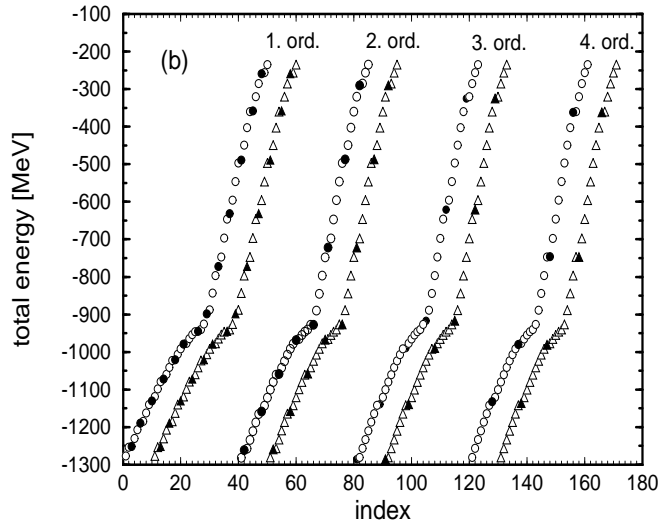

Fig. $5 d$

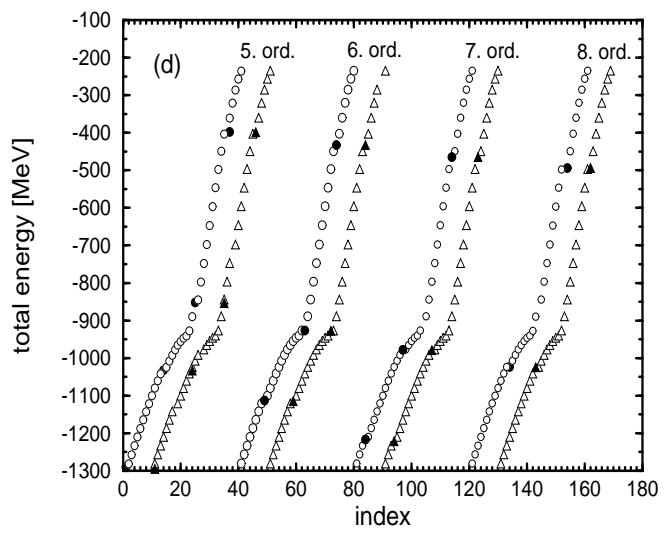



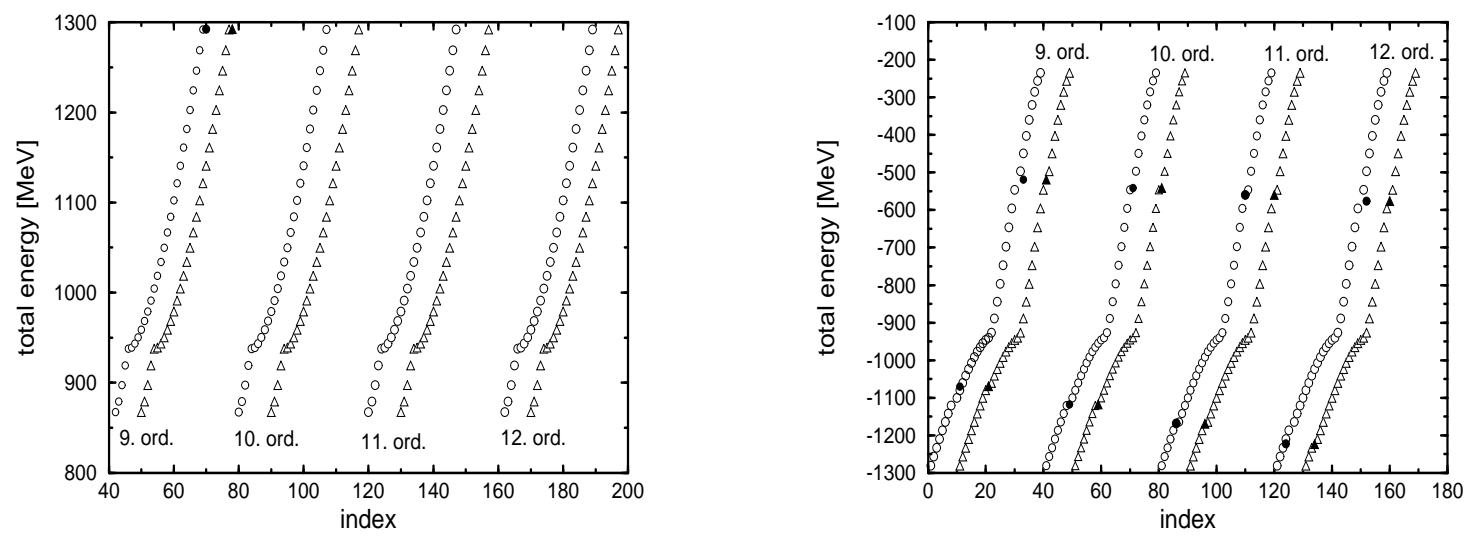

Fig. 5: Energy eigenvalues of the Dirac equation (2) for the case $\kappa=-1$. The spectra are compared for the Lagrange FEM (circles) and the B-spline FEM (triangles). The used finite element orders are indicated in the figures. Filled symbols correspond to spurious eigenvalues. All eigenvalues which appear in the energy window $[-1300 \mathrm{MeV},+1300 \mathrm{MeV}]$ are displayed for $1^{\text {st }}$ order to $12^{\text {th }}$ order elements.

It is also important to analyze the dependence of the spurious spectrum on the number of mesh points. In Fig. 6, the number of spurious solutions is displayed for different orders as a function of the number of mesh points in a constant radial box $\left(r_{\min }=0 \mathrm{fm}, r_{\max }=20 \mathrm{fm}\right)$. The results show that the number of spurious solutions is independent on the number of mesh points if this number is sufficiently large. This is true for all orders of finite elements. The solid lines in Fig. 6 show the results which have been obtained for the above defined Woods-Saxon potentials. For all finite element orders the number of spurious states in the above defined energy window increases at low mesh point numbers and decreases monotonically at high mesh point numbers. At large numbers of mesh points ("asymptotic region") the number of spurious solutions is constant for all element orders. This has been tested up to the very large number of 600 mesh points but is not shown in the figure. For the calculation of the spectra shown in Fig. 5a to 5b, I have used a number of mesh points (200) which is in that asymptotic region to make sure that they (in particular the spurious spectra) are independent on the number of mesh points.

An explanation for the curves in Fig. 6 is found with the concept of Sobolev space. In reference 14] it has been outlined that a Sobolev space $W_{p}^{m}(\Omega)$ is a completion of the test function space $C_{0}^{\infty}(\Omega)$ with respect to the Sobolev norm $\|\cdot\|_{m, p}$ defined in Eq. 23 . Thus, $C_{0}^{\infty}(\Omega) \subset W_{p}^{m}(\Omega)$ for all integer numbers $m \geq 0$. All spaces $W_{p}^{m}(\Omega)$, where $m>0$, are subspace of the largest Sobolev space $W_{p}^{0}(\Omega)$ and

$$
W_{p}^{m+1}(\Omega) \subset W_{p}^{m}(\Omega) \quad \text { for all } \quad m \geq 0 .
$$

The shape functions of $m^{\text {th }}$ order finite elements are element of $W_{p}^{m}(\Omega)$ but the shape functions of any lower order finite elements are not in $W_{p}^{m}(\Omega)$. In finite element discretizations of low order $m$ is small and one works in a correspondingly large space $W_{p}^{m}(\Omega)$. The weak form, of a differential equation, expressed in terms of the weighted residual, allows more solutions than the solutions of the original problem. All solutions which are found for a certain FEM order $m$ are element of $W_{p}^{m}(\Omega)$. For increasing order $m$ the space $W_{p}^{m}(\Omega)$ shrinks and the number of spurious solutions in the weak form is reduced while all physical solutions are maintained. This is seen from Fig. 5a to Fig. 5f for one large number of mesh points. It explains in general the reduction of the number of unphysical solutions in Fig. 6 for all mesh point numbers when the order of the FEM-ansatz is increased. For a uniform finite element mesh with constant width $h$, the $m^{\text {th }}$ order shape functions of the whole FEM discretization span up a space $S_{h}^{m}(\Omega)$. Starting from an initial discretization where $h_{0}$ is large, a sequence of spaces $S_{h_{i}}^{m}(\Omega) i=0,1,2, \ldots$ is generated when the mesh is refined for increasing index $i$, where $h_{i+1}<h_{i}$. The direct sum of the spaces $S_{h_{i}}^{m}(\Omega)$ converges against $W_{p}^{m}(\Omega)$ and thus $\bigoplus_{i=0}^{\infty} S_{h_{i}}^{m}(\Omega)=W_{p}^{m}(\Omega)$. Different spaces $S_{h_{i}}^{m}(\Omega)$ and $S_{h_{j}}^{m}(\Omega)$ where $i \neq j$ can have non-trivial intersection. There are even cases where $S_{h_{i}}^{m}(\Omega) \subset S_{h_{j}}^{m}(\Omega)$ when $h_{i}<h_{j}$. In the example of the two spaces $S_{h}^{1}(\Omega)$ and $S_{h / 2}^{1}(\Omega)$ it is obvious that $S_{h}^{1}(\Omega) \subset S_{h / 2}^{1}(\Omega)$ since each linear shape function 
which is basis function in $S_{h}^{1}(\Omega)$ can be represented as linear combination of shape functions (basis functions) of $S_{h / 2}^{1}(\Omega)$. The strong increase of the graphs in Fig. 6 at small mesh point numbers, where $h$ is large, is explained by the fact that the spaces $S_{h}^{m}(\Omega)$ become large for decreasing $h$. The number of spurious solutions which appear in $S_{h}^{m}(\Omega)$ increases simultaneously. However, there is a second effect which is superposed to this first one.

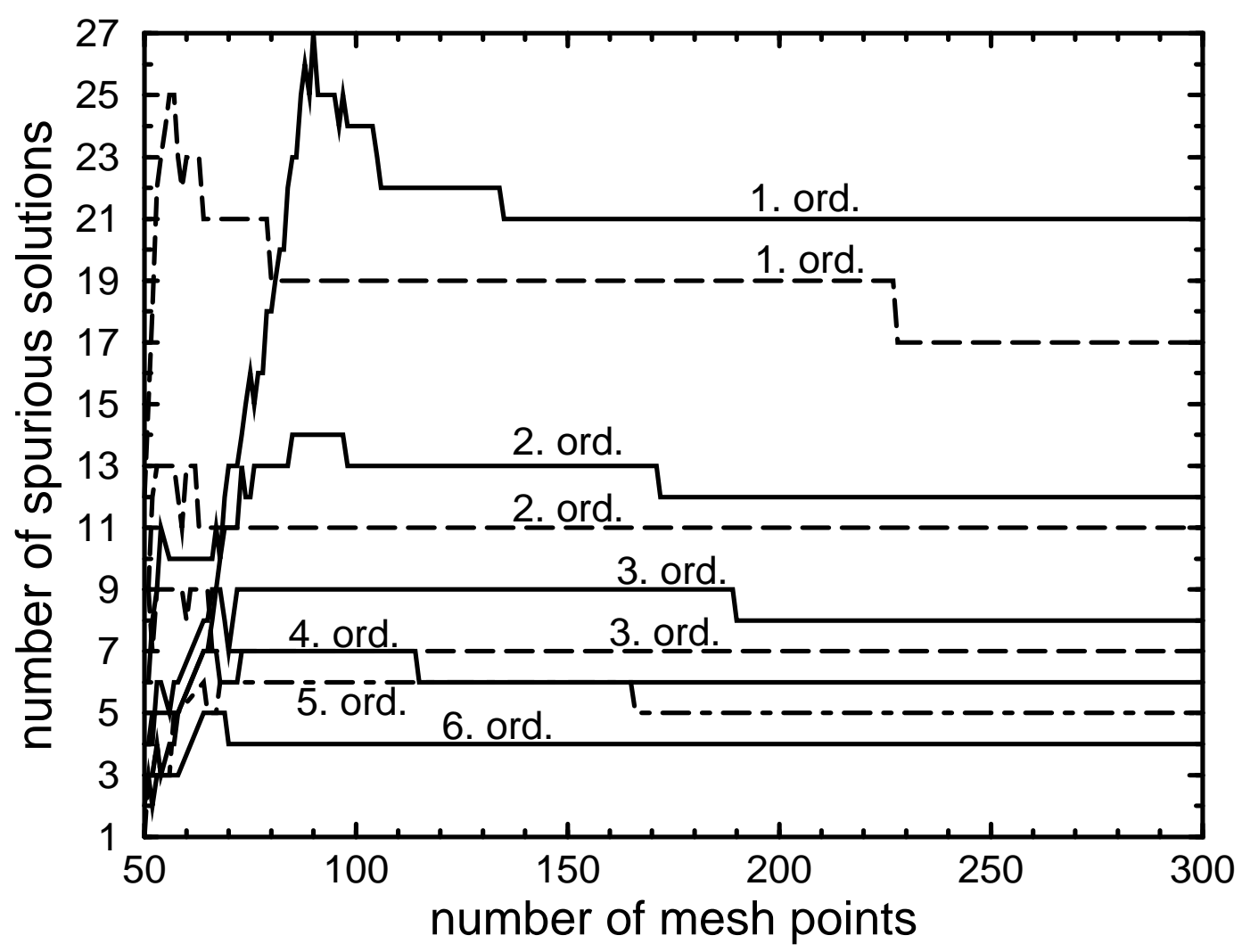

Fig. 6: Dependencies of the number of spurious solutions on the number of mesh points are shown for $1^{\text {st }}$ order to $6^{\text {th }}$ order finite elements. A constant mesh size ranging from $0 \mathrm{fm}$ to $20 \mathrm{fm}$ has been used in the calculations. The solid (and dot-dashed) lines show results obtained for Woods-Saxon potentials $V(r)$ and $S(r)$. The dashed lines show corresponding results for zero potential.

Spurious solutions which have a very high number of oscillations can only be completely resolved in spaces $S_{h}^{m}(\Omega)$ where $h$ is small. However, spurious states with high frequency can appear in subspaces $S_{\tilde{h}}^{m}(\Omega)$ where $\tilde{h}=\nu \cdot h(\nu=1,2,3, \ldots)$ and where only a fraction of the oscillations is resolved. Since the corresponding kinetic energy which contributes to the total energy is small, these solutions appear in the above chosen energy window. If the number of mesh points is increased, additional oscillations are resolved and the kinetic energy increases correspondingly. The corresponding total energy appears no longer in the energy window. In the negative energy range such solutions are shifted further into the negative continuum.

In Fig. 7a and Fig. 7b, spurious energy spectra are displayed for many different mesh point numbers. First order finite elements have been used. The mesh point numbers have been chosen around the maximum of the solid curve in Fig. 6 which corresponds to first order. The solid lines connect spurious eigenvalues which appear at constant mesh point number which is indicated by the numbers atop of each line. 

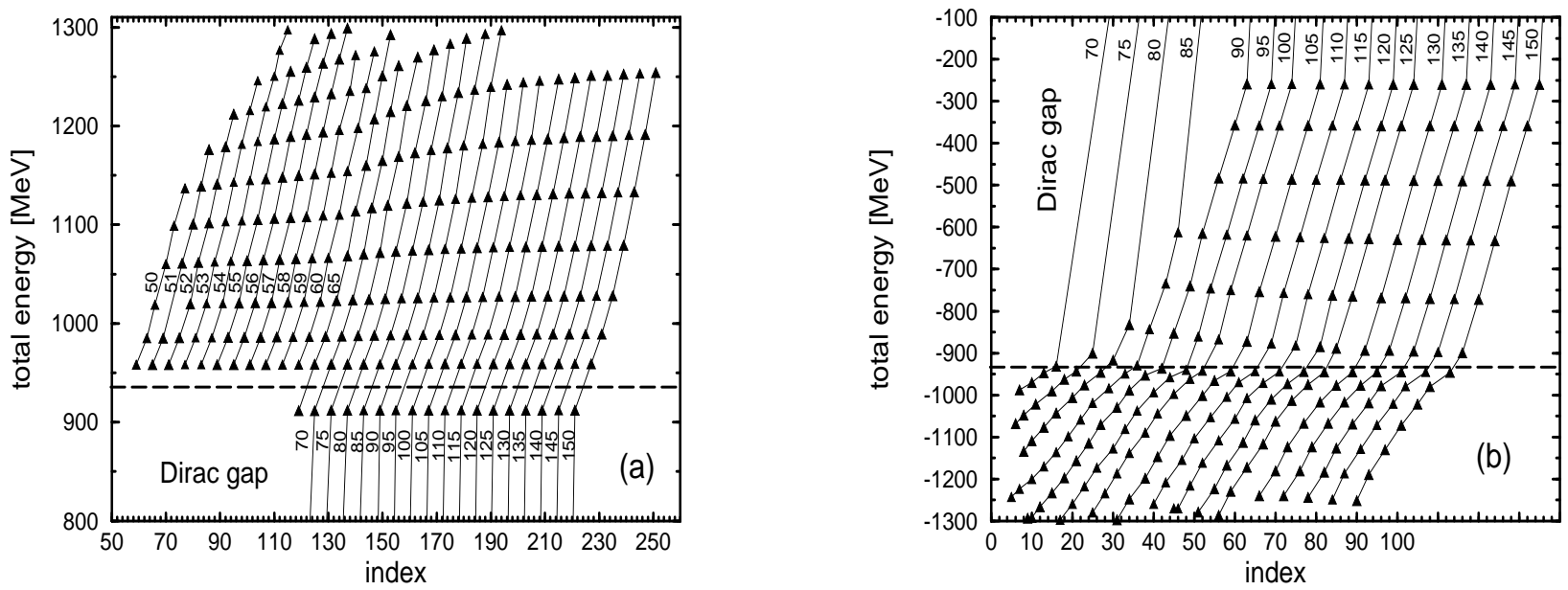

Fig. 7: Spurious spectra of the first order finite element discretization are displayed for different mesh point numbers. The solid lines connect eigenvalues which belong to the same discretization. The number of mesh points is indicated above each line.

\section{NUMERICAL PRECISION}

In the following, I present an analyse of the numerical precision of both methods and compare the results. The quality in the approximation of the exact solutions of (23) depends essentially of the order of the FEM-ansatz and on the number of mesh points used in a given domain $\Omega=\left[r_{\min }, r_{\max }\right]$. In the subsequent tables, neutron single particle eigenvalues are listed systematically for increasing number of mesh points and increasing order of used finite elements. The eigenvalues correspond to solutions which have been obtained in the initial step of a selfconsistent calculation for ${ }^{40} \mathrm{Ca}$. In the first iteration step, Woods-Saxon potentials of the form (41) and (42) have been used with parameters $S(0)=-395 \mathrm{MeV}, V(0)=320 \mathrm{MeV}, a=0.5 \mathrm{fm}$ and $r_{s}=6.0 \mathrm{fm}$. The mesh size has been kept fixed with boundaries at $r_{\min }=0 \mathrm{fm}$ and $r_{\max }=10 \mathrm{fm}$. In Table 1a neutron single particle energies which have been calculated with linear finite elements are shown for the initial WoodsSaxon potential. For increasing number of mesh points (see left column), the number of unchanged decimal places reaches 8 at 200 mesh points. A comparison with the last row of Table 1b shows that for linear elements the last digit (decimal place 10) has not stabilized at the extremely large mesh point number 600 . Table $1 \mathrm{~b}$ displays results that have been calculated with finite elements of $3^{\text {rd }}$ order. Between 109 and 121 mesh points (36-40 elements), the results have stabilized in all 10 digits. In Table 1c, I show the corresponding results which have been obtained with finite elements of $4^{\text {th }}$ order. To demonstrate the enormous improvement in the precision, the results are displayed up to 12 digits. Between 81 and 93 mesh points the $8^{\text {th }}$ digit becomes stable and between 125 and 145 mesh points the precision achieves 12 digits.

\begin{tabular}{|c|c|c|c|c|c|c|}
\hline \multicolumn{7}{|c|}{ 1. Order } \\
\hline$n_{n o d}$ & $E_{1 s 1 / 2}$ & $E_{1 p 3 / 2}$ & $E_{1 p 1 / 2}$ & $E_{1 d 5 / 2}$ & $E_{2 s 1 / 2}$ & $E_{1 d 3 / 2}$ \\
\hline 20 & 65.88133752 & 57.31827220 & 56.39907816 & 47.42408909 & 42.19281200 & 45.28981141 \\
\hline 30 & 65.88108502 & 57.31763456 & 56.39825020 & 47.42279518 & 42.18737854 & 45.28804244 \\
\hline 40 & 65.88104432 & 57.31753150 & 56.39811692 & 47.42258515 & 42.18648990 & 45.28775645 \\
\hline 70 & 65.88102777 & 57.31748952 & 56.39806272 & 47.42249948 & 42.18612708 & 45.28763993 \\
\hline 80 & 65.88102696 & 57.31748747 & 56.39806006 & 47.42249529 & 42.18610928 & 45.28763422 \\
\hline 90 & 65.88102653 & 57.31748638 & 56.39805866 & 47.42249306 & 42.18609982 & 45.28763119 \\
\hline
\end{tabular}




\begin{tabular}{|l|l|l|l|l|r|}
100 & 65.88102628 & 57.31748575 & 56.39805785 & 47.42249178 & 42.18609441 \\
42.18608615 & 45.28762946 \\
150 & 65.88102591 & 57.31748480 & 56.39805662 & 47.42248983 & 47.28762681 \\
200 & 65.88102584 & 57.31748464 & 56.39805641 & 47.42248951 & 42.18608476 \\
45.28762637 \\
300 & 65.88102582 & 57.31748458 & 56.39805634 & 47.42248938 & 42.18608424 \\
600 & 65.88102582 & 57.31748457 & 56.39805632 & 47.42248936 & 42.18608412 \\
\hline
\end{tabular}

Table 1a: Neutron single particle energies in units of $\mathrm{MeV}$ which have been calculated with linear finite elements of Lagrange type. The number of used mesh points has been gradually increased as shown in the left column.

\begin{tabular}{|c|c|c|c|c|c|c|}
\hline \multicolumn{7}{|c|}{ 3. Order } \\
\hline$n_{\text {nod }}$ & $E_{1 s 1 / 2}$ & $E_{1 p 3 / 2}$ & $E_{1 p 1 / 2}$ & $E_{1 d 5 / 2}$ & $E_{2 s 1 / 2}$ & $E_{1 d 3 / 2}$ \\
\hline 19 & 65.88080395 & 57.31691348 & 56.39762680 & 47.42131836 & 42.18517027 & 45.28703195 \\
\hline 25 & 65.88103067 & 57.31748586 & 56.39806263 & 47.42248096 & 42.18636302 & 45.28761163 \\
\hline 31 & 65.88103176 & 57.31749678 & 56.39809005 & 47.42250968 & 42.18614523 & 45.28768121 \\
\hline 52 & 65.88102587 & 57.31748468 & 56.39805656 & 47.42248956 & 42.18608460 & 45.28762657 \\
\hline 61 & 65.88102583 & 57.31748460 & 56.39805637 & 47.42248941 & 42.18608424 & 45.28762624 \\
\hline 70 & 65.88102582 & 57.31748458 & 56.39805633 & 47.42248937 & 42.18608415 & 45.28762619 \\
\hline 79 & 65.88102582 & 57.31748457 & 56.39805632 & 47.42248936 & 42.18608413 & 45.28762617 \\
\hline 91 & 65.88102582 & 57.31748457 & 56.39805632 & 47.42248936 & 42.18608412 & 45.28762616 \\
\hline
\end{tabular}

Table 1b: $\quad$ Same as Table 1a but for Lagrange FEM of $3^{\text {rd }}$ order. 


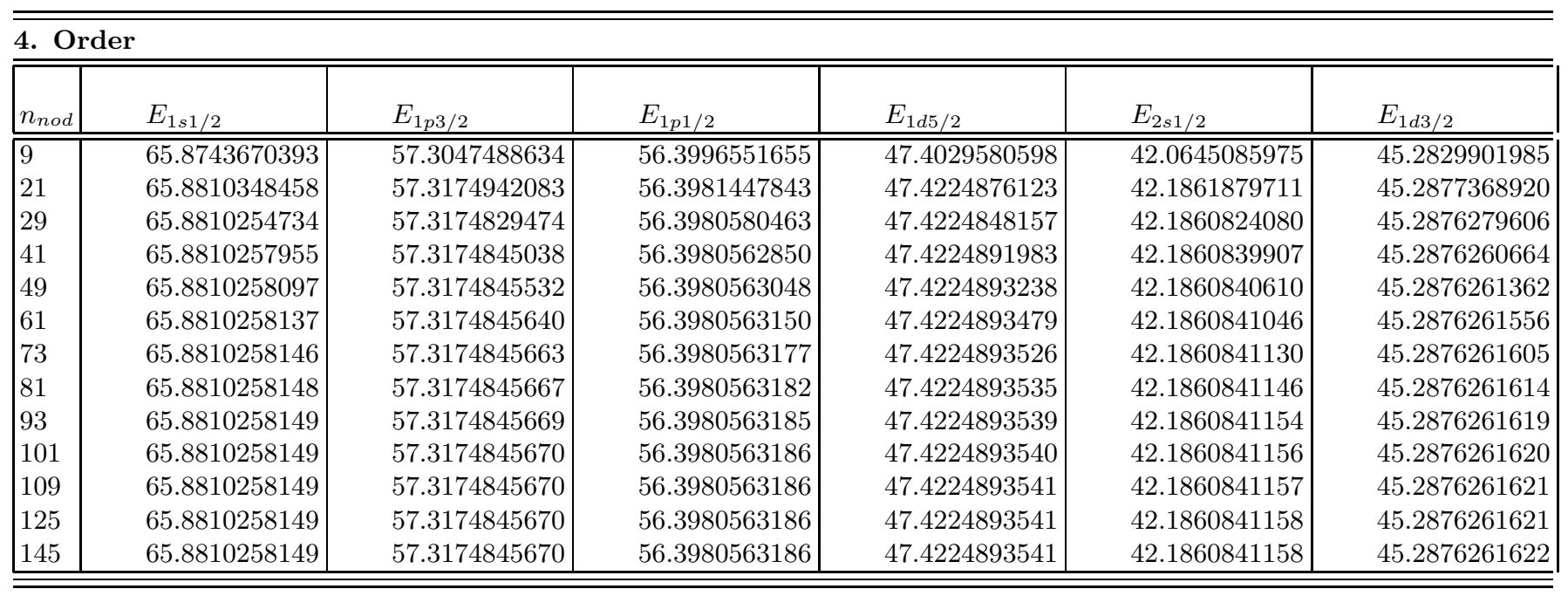

Table 1c: $\quad$ Same as Table $1 \mathrm{~b}$ but for $4^{\text {th }}$ order Lagrange FEM.

\begin{tabular}{l|l|l|l|l|r|r|}
\hline \hline 5. Order \\
\hline \hline & & & & & \\
$n_{\text {nod }}$ & $E_{1 s 1 / 2}$ & $E_{1 p 3 / 2}$ & $E_{1 p 1 / 2}$ & $E_{1 d 5 / 2}$ & $E_{2 s 1 / 2}$ & $E_{1 d 3 / 2}$ \\
\hline \hline 11 & 65.8784571165 & 57.3086583184 & 56.3940420670 & 47.4017253841 & 42.1771582984 & 45.2818654596 \\
21 & 65.8810087294 & 57.3174496620 & 56.3980829394 & 47.4224368621 & 42.1858924489 & 45.2876670981 \\
31 & 65.8810255741 & 57.3174839393 & 56.3980557441 & 47.4224881501 & 42.1860830079 & 45.2876254614 \\
41 & 65.8810258056 & 57.3174845338 & 56.3980562769 & 47.4224892693 & 42.1860841170 & 45.2876260714 \\
51 & 65.8810258150 & 57.3174845669 & 56.3980563285 & 47.4224893538 & 42.1860841175 & 45.2876261744 \\
61 & 65.8810258146 & 57.3174845661 & 56.3980563217 & 47.4224893521 & 42.1860841144 & 45.2876261671 \\
71 & 65.8810258149 & 57.3174845668 & 56.3980563189 & 47.4224893537 & 42.1860841158 & 45.2876261626 \\
81 & 65.8810258149 & 57.3174845670 & 56.3980563187 & 47.4224893541 & 42.1860841159 & 45.2876261622 \\
\hline \hline
\end{tabular}

Table 1d: Same as Table 1d but for $5^{\text {th }}$ order Lagrange FEM. 


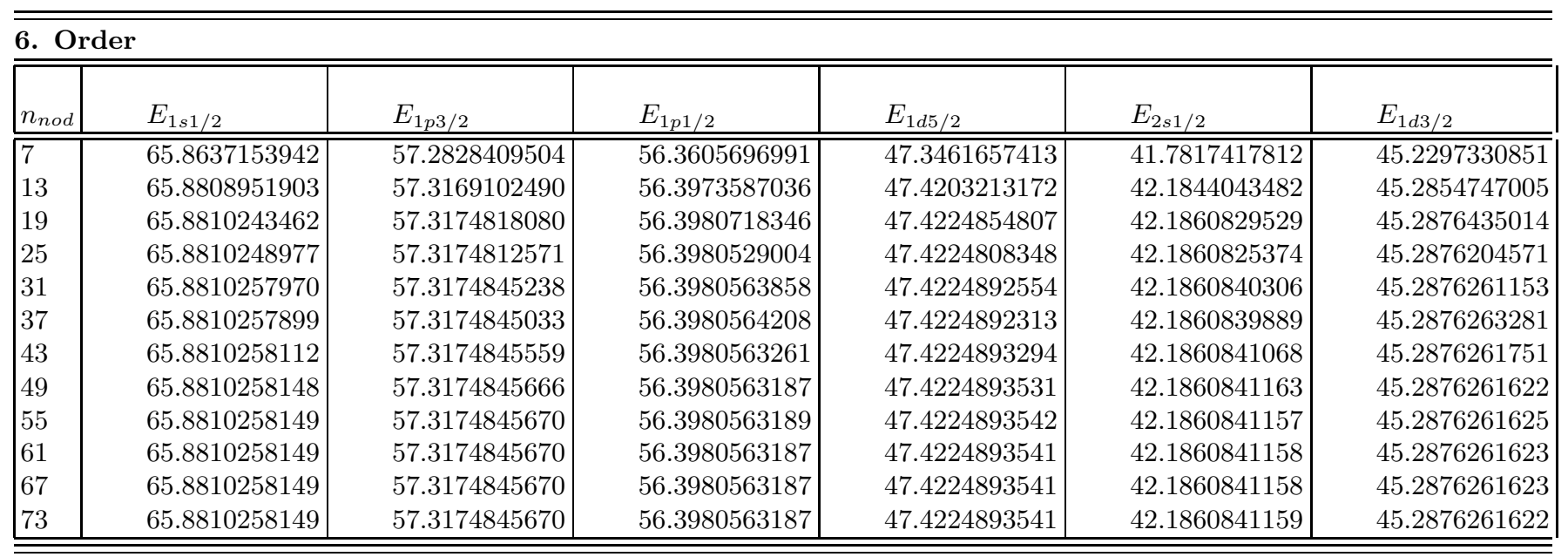

Table 1e: Same as Table 1d but for $6^{\text {th }}$ order Lagrange FEM.

\begin{tabular}{|c|c|c|c|c|c|c|}
\hline \multicolumn{7}{|c|}{ 7. Order } \\
\hline$n_{\text {nod }}$ & $E_{1 s 1 / 2}$ & $E_{1 p 3 / 2}$ & $E_{1 p 1 / 2}$ & $E_{1 d 5 / 2}$ & $E_{2 s 1 / 2}$ & $E_{1 d 3 / 2}$ \\
\hline 15 & 65.8808831962 & 57.3171483037 & 56.3981295386 & 47.4219539180 & 42.1850478466 & 45.2877364182 \\
\hline 22 & 65.8810247273 & 57.3174817834 & 56.3980551153 & 47.4224839062 & 42.1860817524 & 45.2876261615 \\
\hline 29 & 65.8810255033 & 57.3174838576 & 56.3980570146 & 47.4224881798 & 42.1860821285 & 45.2876272423 \\
\hline 50 & 65.8810258149 & 57.3174845670 & 56.3980563186 & 47.4224893540 & 42.1860841156 & 45.2876261621 \\
\hline 57 & 65.8810258149 & 57.3174845670 & 56.3980563187 & 47.4224893541 & 42.1860841158 & 45.2876261622 \\
\hline 64 & 65.8810258149 & 57.3174845670 & 56.3980563187 & 47.4224893541 & 42.1860841158 & 45.2876261622 \\
\hline 71 & 65.8810258149 & 57.3174845670 & 56.3980563187 & 47.4224893541 & 42.1860841159 & 45.2876261622 \\
\hline
\end{tabular}

Table 1f: Same as Table 1e but for $7^{\text {th }}$ order Lagrange FEM. 
Table 1d displays eigenvalues which have been calculated with finite elements of $5^{\text {th }}$ order. At 76 mesh points 12 digits have stabilized for all 6 eigenvalues. At 41 mesh points the precision is already as good as the precision in Table 1a at 600 mesh points. A comparison of the eigenvalues in Table $1 \mathrm{~d}$ with results of a $6^{\text {th }}$ order FEM calculation in Table 1e shows that a further increase of the order leads to a rather weak reduction of the number of required mesh points. At least 73 mesh points are necessary in $6^{\text {th }}$ order for a precision of 12 digits. As shown in Table $1 \mathrm{f}$, the reduction of the number of mesh points is even weaker when the order is increased from $6^{\text {th }}$ order to $7^{\text {th }}$ order. In the subsequent Tables 2a to $2 \mathrm{f}$, results of corresponding calculations with B-spline finite elements are shown. In Table 2a, neutron single particle eigenvalues are listed which have been calculated with the new B-spline FEM code. A comparison of the numbers with those listed in Table 1a shows that they are identical for equal mesh point numbers. For increasing order of the B-splines, the number of required mesh points to obtain a certain precision reduces very similarly to the trend observed in the Tables 1a to 1f. A comparison of the Tables 2a to $2 \mathrm{f}$ with the corresponding Tables 1a to 1f shows that roughly half the number of mesh points is required in a B-spline FEM in order to achieve the precision of a corresponding calculation with Lagrangian finite elements. In Table 1b, full precision is achieved at 60 mesh points while 121 mesh points were necessary in Table $1 \mathrm{~b}$. In a calculation with $4^{\text {th }}$ order B-spline elements, 45 mesh points are required as shown in Table 2c whereas 145 mesh points are necessary with Lagrange elements (Table 1c) to obtain a precision of 12 digits. In the $5^{\text {th }}$ order B-spline FEM, 34 mesh points have been used (Table $2 \mathrm{~d}$ ) while a corresponding $5^{\text {th }}$ order Langrange FEM required 76 mesh points (Table 1d). The $6^{\text {th }}$ order B-spline FEM (see results in Table 2e) leads still to a considerable relative reduction of the number of mesh points from 34 to 30 at the same level of precision while in the $7^{\text {th }}$ order method still 29 mesh points were required (Table 2f). The results shown in the Tables $1 \mathrm{a}$ to $1 \mathrm{f}$ and in the Tables $2 \mathrm{a}$ to $2 \mathrm{f}$ lead to the conclusion that the B-spline FEM has its optimum at $6^{\text {th }}$ order whereas the optimal order of the Lagrange FEM is at $5^{\text {th }}$ order. However, the optimal order may depend on the required precision.

\begin{tabular}{|c|c|c|c|c|c|c|}
\hline \multicolumn{7}{|c|}{ 1. Order } \\
\hline$n_{\text {nod }}$ & $E_{1 s 1 / 2}$ & $E_{1 p 3 / 2}$ & $E_{1 p 1 / 2}$ & $E_{1 d 5 / 2}$ & $E_{2 s 1 / 2}$ & $E_{1 d 3 / 2}$ \\
\hline 20 & 65.88133752 & 57.31827220 & 56.39907816 & 47.42408909 & 42.19281200 & 45.28981141 \\
\hline 30 & 65.88108502 & 57.31763456 & 56.39825020 & 47.42279518 & 42.18737854 & 45.28804244 \\
\hline 40 & 65.88104432 & 57.31753150 & 56.39811692 & 47.42258515 & 42.18648990 & 45.28775645 \\
\hline 70 & 65.88102777 & 57.31748952 & 56.39806272 & 47.42249948 & 42.18612708 & 45.28763993 \\
\hline 80 & 65.88102696 & 57.31748747 & 56.39806006 & 47.42249529 & 42.18610928 & 45.28763422 \\
\hline 90 & 65.88102653 & 57.31748638 & 56.39805866 & 47.42249306 & 42.18609982 & 45.28763119 \\
\hline 100 & 65.88102628 & 57.31748575 & 56.39805785 & 47.42249178 & 42.18609441 & 45.28762946 \\
\hline 150 & 65.88102591 & 57.31748480 & 56.39805662 & 47.42248983 & 42.18608615 & 45.28762681 \\
\hline
\end{tabular}

Table 2a: Same as Table 1a but for B-spline FEM. 


\begin{tabular}{|c|c|c|c|c|c|c|}
\hline \multicolumn{7}{|c|}{ 3. Order } \\
\hline$n_{\text {nod }}$ & $E_{1 s 1 / 2}$ & $E_{1 p 3 / 2}$ & $E_{1 p 1 / 2}$ & $E_{1 d 5 / 2}$ & $E_{2 s 1 / 2}$ & $E_{1 d 3 / 2}$ \\
\hline 20 & 65.88102627 & 57.31748559 & 56.39805814 & 47.42249117 & 42.18608805 & 45.28762926 \\
\hline 30 & 65.88102583 & 57.31748459 & 56.39805636 & 47.42248941 & 42.18608422 & 45.28762623 \\
\hline 40 & 65.88102582 & 57.31748457 & 56.39805632 & 47.42248936 & 42.18608413 & 45.28762617 \\
\hline 70 & 65.88102581 & 57.31748457 & 56.39805632 & 47.42248935 & 42.18608412 & 45.28762616 \\
\hline
\end{tabular}

Table 2b: Same as Table 1b but for B-spline FEM.

\begin{tabular}{|c|c|c|c|c|c|c|}
\hline \multicolumn{7}{|c|}{ 4. Order } \\
\hline$n_{\text {nod }}$ & $E_{1 s 1 / 2}$ & $E_{1 p 3 / 2}$ & $E_{1 p 1 / 2}$ & $E_{1 d 5 / 2}$ & $E_{2 s 1 / 2}$ & $E_{1 d 3 / 2}$ \\
\hline 7 & 65.87919142 & 57.31238878 & 56.39343856 & 47.41226119 & 42.18527059 & 45.28088247 \\
\hline 8 & 65.88036297 & 57.31616474 & 56.40144465 & 47.42037951 & 42.18049023 & 45.29330556 \\
\hline 9 & 65.88118037 & 57.31768604 & 56.39894718 & 47.42267251 & 42.18838460 & 45.28845841 \\
\hline 20 & 65.88102585 & 57.31748464 & 56.39805656 & 47.42248949 & 42.18608449 & 45.28762653 \\
\hline 25 & 65.88102582 & 57.31748457 & 56.39805633 & 47.42248937 & 42.18608414 & 45.28762618 \\
\hline 30 & 65.88102582 & 57.31748457 & 56.39805632 & 47.42248936 & 42.18608412 & 45.28762616 \\
\hline 35 & 65.8810258150 & 57.3174845672 & 56.3980563189 & 47.4224893544 & 42.1860841164 & 45.2876261626 \\
\hline 40 & 65.8810258149 & 57.3174845670 & 56.3980563187 & 47.4224893542 & 42.1860841160 & 45.2876261623 \\
\hline
\end{tabular}

Table 2c: Same as Table 1c but for B-spline FEM.

\begin{tabular}{|c|c|c|c|c|c|c|}
\hline \multicolumn{7}{|c|}{ 5. Order } \\
\hline$n_{\text {nod }}$ & $E_{1 s 1 / 2}$ & $E_{1 p 3 / 2}$ & $E_{1 p 1 / 2}$ & $E_{1 d 5 / 2}$ & $E_{2 s 1 / 2}$ & $E_{1 d 3 / 2}$ \\
\hline 10 & 65.88103149 & 57.31749002 & 56.39852712 & 47.42249777 & 42.18607033 & 45.28816996 \\
\hline 20 & 65.88102581 & 57.31748455 & 56.39805651 & 47.42248931 & 42.18608408 & 45.28762647 \\
\hline 30 & 65.8810258149 & 57.3174845671 & 56.3980563188 & 47.4224893542 & 42.1860841160 & 45.2876261624 \\
\hline 33 & 65.8810258149 & 57.3174845670 & 56.3980563187 & 47.4224893542 & 42.1860841159 & 45.2876261622 \\
\hline 34 & 65.8810258149 & 57.3174845670 & 56.3980563187 & 47.4224893541 & 42.1860841159 & 45.2876261622 \\
\hline
\end{tabular}

Table 2d: Same as Table 1d but for B-spline FEM. 


\begin{tabular}{|c|c|c|c|c|c|c|}
\hline \multicolumn{7}{|c|}{ 6. Order } \\
\hline$n_{n o d}$ & $E_{1 s 1 / 2}$ & $E_{1 p 3 / 2}$ & $E_{1 p 1 / 2}$ & $E_{1 d 5 / 2}$ & $E_{2 s 1 / 2}$ & $E_{1 d 3 / 2}$ \\
\hline 15 & 65.88102458 & 57.31748100 & 56.39805735 & 47.42248186 & 42.18608340 & 45.28762800 \\
\hline 20 & 65.8810258052 & 57.3174845391 & 56.3980563477 & 47.4224892980 & 42.1860841341 & 45.2876262023 \\
\hline 25 & 65.8810258149 & 57.3174845669 & 56.3980563193 & 47.4224893539 & 42.1860841164 & 45.2876261632 \\
\hline 28 & 65.8810258149 & 57.3174845670 & 56.3980563188 & 47.4224893541 & 42.1860841159 & 45.2876261623 \\
\hline 29 & 65.8810258149 & 57.3174845670 & 56.3980563187 & 47.4224893541 & 42.1860841159 & 45.2876261623 \\
\hline 30 & 65.8810258149 & 57.3174845670 & 56.3980563187 & 47.4224893541 & 42.1860841159 & 45.2876261622 \\
\hline
\end{tabular}

Table 2e: Same as Table 1e but for B-spline FEM.

\begin{tabular}{|c|c|c|c|c|c|c|}
\hline \multicolumn{7}{|c|}{ 7. Order } \\
\hline$n_{\text {nod }}$ & $E_{1 s 1 / 2}$ & $E_{1 p 3 / 2}$ & $E_{1 p 1 / 2}$ & $E_{1 d 5 / 2}$ & $E_{2 s 1 / 2}$ & $E_{1 d 3 / 2}$ \\
\hline 15 & 65.88102496 & 57.31748290 & 56.39806113 & 47.42248693 & 42.18607611 & 45.28763254 \\
\hline 20 & 65.88102580 & 57.31748453 & 56.39805640 & 47.42248929 & 42.18608399 & 45.28762629 \\
\hline 25 & 65.8810258147 & 57.3174845665 & 56.3980563197 & 47.4224893532 & 42.1860841144 & 45.2876261638 \\
\hline 28 & 65.8810258149 & 57.3174845670 & 56.3980563187 & 47.4224893541 & 42.1860841158 & 45.2876261622 \\
\hline 29 & 65.8810258149 & 57.3174845670 & 56.3980563187 & 47.4224893541 & 42.1860841159 & 45.2876261622 \\
\hline
\end{tabular}

Table 2f: $\quad$ Same as Table 1f but for B-spline FEM. 
To complete this study, I repeated the calculations for a large number of mesh points with both methods from $1^{\text {st }}$ order to $8^{\text {th }}$ order finite elements. In the figures Fig. 8a to Fig. 8d, the logarithmic errors with respect to the highest precision are shown. Fig. 8a displays the averaged error taken over all 6 single particle energies which have been calculated with the B-spline FEM. In Fig. 8b, these data have been smoothed by taking in addition the average over two neighboring mesh point numbers. In the region of precision ranging from $10^{-1}$ to $10^{-10.5}$, both figures display an enormous reduction of the errors for increasing finite element order up to $5^{\text {th }}$ order. Finite elements of higher order do not essentially improve the precision in the considered range but entail a higher numerical cost. They may improve the precision beyond the error range of $\left[10^{-1}, 10^{-10}\right]$. However, precisions in the range of $\left[10^{-1}, 10^{-10}\right]$ are sufficient in most applications. In Fig. $8 \mathrm{~b}$ there is an indication for $6^{\text {th }}$ order to become optimal order at precisions better than $10^{-10}$. This is in agreement with the conclusion that has been drawn form the data in Table $2 \mathrm{a}$ to $2 \mathrm{f}$

In Fig. 8c and Fig. 8d. results that correspond to Fig. 8a and Fig. 8b but calculated with the Lagrange FEM are displayed. A similar but weaker reduction of the errors is observed for increasing finite element order. A comparison with the results depicted in Fig. 8a and Fig. 8b shows that the B-spline method requires in general a much smaller number of mesh points than the Lagrange FEM in order to provide the desired level of precision. For comparison, in Fig. 8d, I have inserted those graphs of Fig. 8b which resulted for $5^{\text {th }}$ order to $8^{\text {th }}$ order B-spline calculations.
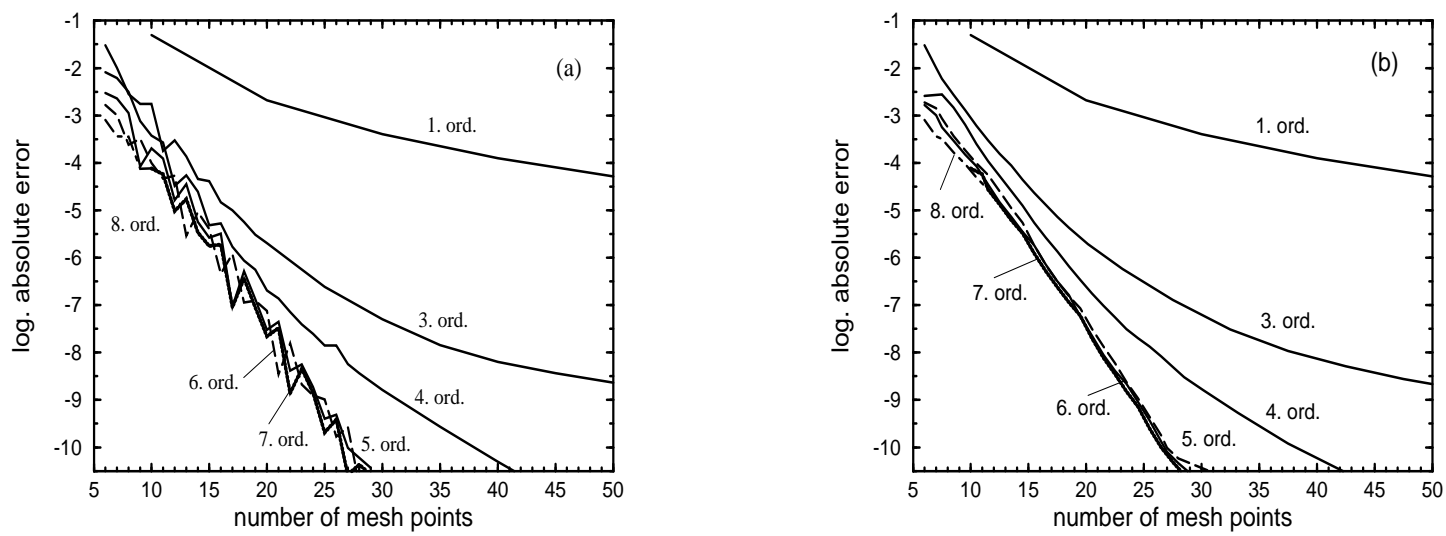

Fig. $8 \mathrm{c}$
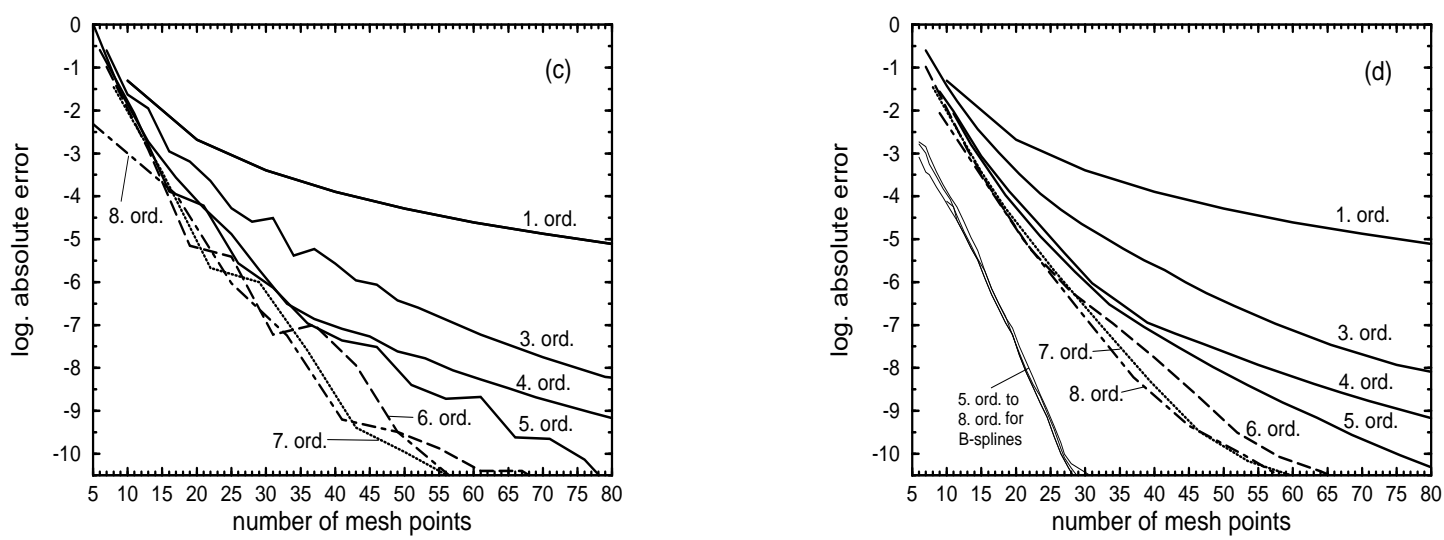

Fig. 8: Logarithmic plots of the error which occurs in the B-spline FEM (figures (a),(b)) and in the Lagrange FEM (figures (c),(d)). The used finite element orders are indicated. Figs. (a) and (c) display average values taken over the six lowest positive neutron single particle eigenvalues. Figs. (b) and (d) show the smoothed curves. 


\section{PERFORMANCE DISCUSSION}

With respect to applications of the above presented B-spline FEM in large scale computations with FEM discretizations in more than one dimension, attention should be payed to the performance of both methods at the present stage. Therefore, I investigate and compare the run time for both methods, the B-spline FEM and the Lagrange FEM. The following discussion is based on data which correspond to the performance of the codes on a DEC Alpha $300 \mathrm{MHz}$. A gnu compiler has been used under UNIX to translate the codes.

The CPU-time depends essentially on the FEM order and the number of used mesh points. The times which are displayed in the Figs. $9 \mathrm{a}$ and $9 \mathrm{~b}$ correspond to a single step in which the Dirac equation 2 is solved. This procedure encloses essentially the construction and the solution of the generalized eigenvalue problem for one $\kappa$-value. It has to be repeated for each $\kappa$ in the solver for neutron and proton states and this again over the whole self-consistent iteration. The CPU-time which is required for the solution of the meson field equations lies below one percent of that for the nucleon states and is therefore neglected. Fig. 9a displays the CPU-time for $5^{\text {th }}$ order FEM as a function of the number of mesh points. Results which have been obtained for other orders are almost identical with those shown in the figure. The solid curve displays CPU-times resulting from Lagrange type finite element discretizations while the dashed curve has been obtained with the B-spline FEM. An explanation for the higher numerical cost in applications of the B-spline method is given by Figs. 4 showing that more matrix elements have to be calculated in the case of B-spline FEM.

However, as demonstrated in the Figs. 8, the number of required mesh points for equal numerical precision is half of that in the Lagrange FEM. At equal numerical precision, the solid curve has to be compared with the dot-dashed curve of a B-spline calculation. This clearly demonstrates an enormous reduction of the numerical cost for the B-spline FEM.

In Fig. 9b, CPU-times are plotted as a function of the FEM order and compared for both methods. The precision of the numerical solution which has been obtained with the B-spline FEM (dashed line) is much higher that that obtained with the Lagrange FEM (solid line). At equal numerical precision, one should compare values of the dashed line with values of the solid line at double order.

Fig. $9 a$
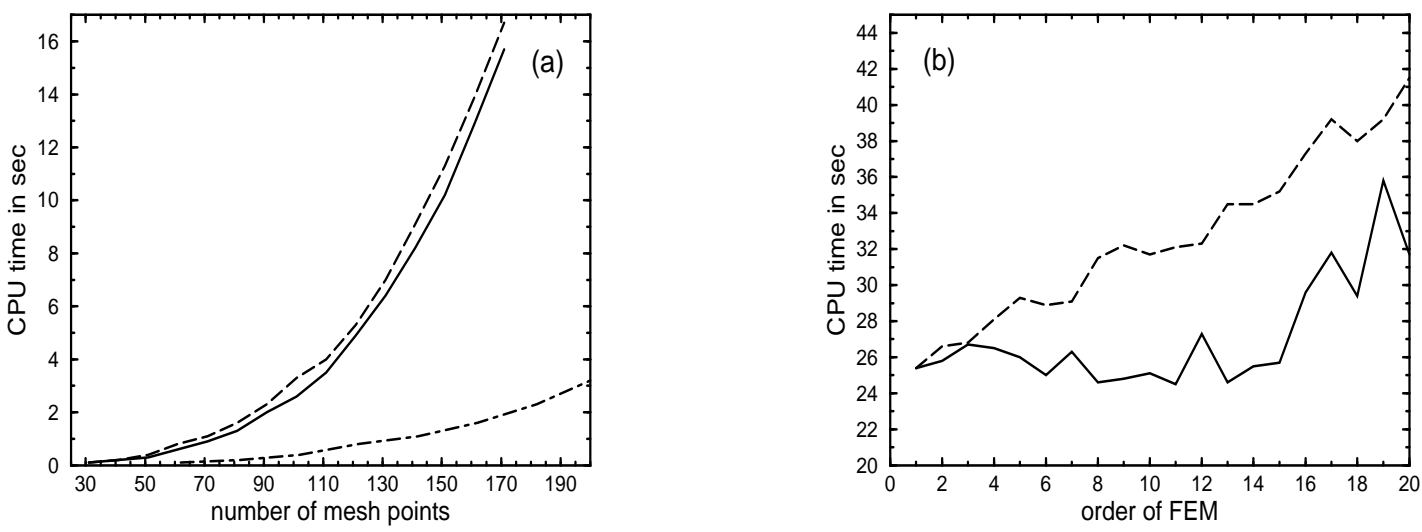

Fig. 9: CPU time as a function of the number of used mesh points (a) and as a function of used order (b) for both methods, B-spline FEM and Lagrange FEM.

\section{PROGRAM STRUCTURE}

The program is coded in $\mathrm{C}++$. The implementation of the relativistic mean field model in the Hartree approximation for spherical doubly-closed shell nuclei has been described in Ref. 14. In this section we only describe the changes that have been made in order to modify the program to B-spline techniques.

The main part of the program consists of seven classes: MathPar: numerical parameters used in the code, PhysPar: physical parameters (masses, coupling constants, etc.), FinEl: finite ele- 
ments, Mesh: mesh in coordinate space, Nucleon: neutrons and protons in the nuclear system, Meson: mesons and photon with corresponding mean fields and the Coulomb field, and the class LinBCGOp. A detailed description of these classes can be found in Ref. 14].

Two new classes FinElBsp and BSpline have been added to the code. The implementation can be found in the source files finelbsp.cc, bspline.cc and the corresponding header files finelbsp.h, bspline.h. The class FinElBsp contains the following new member functions:

FinElBsp();

$\tilde{F} i n \operatorname{ElBsp}()$;

void FinElBsp::alloc( int ord );

void FinElBsp::free();

void FinElBsp::make( int ord );

double FinElBsp::n( int iloc, int ife, int iga, int l, bool zero ) const;

double FinElBsp::dn( int iloc, int ife, int iga, int l, bool zero ) const;

double FinElBsp::func( double const* ${ }^{*}$, int ife, int iga) const;

double FinElBsp::func( double const* ${ }^{*}$,int ife,int iga,int l,bool zero ) const;

void FinElBsp::eval();

inline int FinElBsp::order() const;

inline int FinElBsp::nloc() const;

inline double FinElBsp::n( int iloc, int iga) const;

inline double FinElBsp::dn( int iloc, int iga ) const;

inline double FinElBsp::operator()(double const* ${ }^{*}$,int ife,int iga) const;

inline double FinElBsp::operator()(double const* ${ }^{*}$,int ife,int iga,int l, bool zero ) const; The two methods FinElBsp() and FinElBsp() describe the constructor and destructor of objects (B-spline finite elements) of the class. The method make( int ord) provids the B-spline reference element with data (amplitudes) on the shape functions and their derivatives. Access to these data is given through the methodes $n$ (int iloc, int iga) and $d n$ (int iloc, int iga). In a first step, make (int ord) allocates memory for the shape functions using alloc(int ord). In a second step, the method eval() is called generating the amplitudes of the shape functions through an operator of class BSpline - The overload member functions $n$ (int iloc, int ife, int iga, int l, bool zero ) const and $d n$ (int iloc, int ife, int iga, int l, bool zero ) const are used in the calculation of the stiffness matrices. They take into account boundary conditions. The method func(double const* $u$, int ife, int iga) const provides the interpolated amplitude of solutions on the Gauss submesh in any finite element of global index ife. The overloaded version func(double const* $u$, int ife, int iga) const takes boundary conditions into account.

In the class BSpline, the following methods are implemented BSpline( int ord) BSSpline() operator()( double const* ${ }^{*}$, double\& $f$, double\& $d f$, double $x$ ) inline int BSpline::order() const;

BSpline( int ord) and $\tilde{B}$ Spline() describe the constructor and the destructor of the class. An operator is used to carry out the B-spline algorithm (22) whenever access to B-spline amplitudes is requested through a call of an object of the class with corresponding arguments.

The organization in the construction of stiffness matrices in other parts of the code has been changed accordingly. However, the essential structure has been maintained so that quick changes for applications of Langrange type finite elements (defined in class FinEl) are possible. An essential difference roots in the relation between number of nodes on the global mesh and the number of finite elements which is given by $n^{\text {nod }}=n^{\text {fe }}+n^{\text {ord }}$. In the version using Langrange type elements this relation is $n^{\text {nod }}=n^{\text {fe }} \cdot n^{\text {ord }}+1$.

For the diagonalization of the generalized eigenvalue problems the bisection method has been replaced by a combined Cholesky decomposition and householder method which is slower but allows for a higher precision. A heapsort algorithm orders eigenvalues and eigenvectors. The routines and the eigensolver are implemented in the source file eigen.cc and the header file eigen.h. 


\section{APPENDIX: APPENDIX}

For the FEM discretization of the Klein-Gordon equations we use the ansatz

$$
\begin{aligned}
\sigma(r) & =\sum_{p} \sigma_{p} B_{p}(r) \\
\omega^{0}(r) & =\sum_{p} \omega_{p}^{0} B_{p}(r) \\
\rho^{0}(r) & =\sum_{p} \rho_{p}^{0} B_{p}(r) \\
A^{0}(r) & =\sum_{p} A_{p}^{0} B_{p}(r) .
\end{aligned}
$$

where the node variables $\sigma_{p}, \omega_{p}^{0}, \rho_{p}^{0}$ and $A_{p}^{0}$ correspond to field amplitudes at the mesh point $\mathrm{p}$. For the Klein-Gordon equations we use the same type of shape functions $B_{p}(r)$, and the same mesh as in the FEM discretization of the Dirac equation (24). Using again the method of weighted residuals with test functions $w_{p}(r)=r^{2} B_{p}(r)$, the following algebraic equations are obtained

$$
\begin{gathered}
\sum_{p}\left\langle w_{p^{\prime}}(r)\left|-\partial_{r}^{2}-\frac{2}{r} \partial_{r}+\frac{l(l+1)}{r^{2}}+m_{\sigma}^{2}\right| B_{p}(r)\right\rangle \sigma_{p}=\left\langle w_{p^{\prime}}(r) \mid s_{\sigma}\left(\Phi_{1}(r), \ldots, \Phi_{A}(r)\right)\right\rangle \\
\sum_{p}\left\langle w_{p^{\prime}}(r)\left|-\partial_{r}^{2}-\frac{2}{r} \partial_{r}+\frac{l(l+1)}{r^{2}}+m_{\omega}^{2}\right| B_{p}(r)\right\rangle \omega_{p}^{0}=\left\langle w_{p^{\prime}}(r) \mid s_{\omega}\left(\Phi_{1}(r), \ldots, \Phi_{A}(r)\right)\right\rangle \\
\sum_{p}\left\langle w_{p^{\prime}}(r)\left|-\partial_{r}^{2}-\frac{2}{r} \partial_{r}+\frac{l(l+1)}{r^{2}}+m_{\rho}^{2}\right| B_{p}(r)\right\rangle \rho_{p}^{0}=\left\langle w_{p^{\prime}}(r) \mid s_{\rho}\left(\Phi_{1}(r), \ldots, \Phi_{A}(r)\right)\right\rangle \\
\sum_{p}\left\langle w_{p^{\prime}}(r)\left|-\partial_{r}^{2}-\frac{2}{r} \partial_{r}+\frac{l(l+1)}{r^{2}}\right| B_{p}(r)\right\rangle A_{p}^{0}=\left\langle w_{p^{\prime}}(r) \mid s_{C}\left(\Phi_{1}(r), \ldots, \Phi_{A}(r)\right)\right\rangle .
\end{gathered}
$$

The resulting matrix equations read

$$
\begin{aligned}
{\left[S_{1}^{\sigma}+l(l+1) \cdot S_{2}^{\sigma}+m_{\sigma}^{2} \cdot S_{3}^{\sigma}+S_{4}^{\sigma}\right] \vec{\sigma} } & =\vec{r}^{(\mathrm{s})} \\
{\left[S_{1}^{\omega}+l(l+1) \cdot S_{2}^{\omega}+m_{\omega}^{2} \cdot S_{3}^{\omega}\right] \cdot \vec{\omega}^{0} } & =\vec{r}^{(\mathrm{v})} \\
{\left[S_{1}^{\rho}+l(l+1) \cdot S_{2}^{\rho}+m_{\rho}^{2} \cdot S_{3}^{\rho}\right] \cdot \vec{\rho}^{0} } & =\vec{r}^{(3)} \\
{\left[S_{1}^{A^{0}}+l(l+1) \cdot S_{2}^{A^{0}}\right] \cdot \vec{A}^{0} } & =\vec{r}^{(\mathrm{em})} .
\end{aligned}
$$

The node variables $\sigma_{p}, \omega_{p}^{0}, \rho_{p}^{0}$ and $A_{p}^{0}$ are grouped into the vectors $\vec{\sigma}=\left(\sigma_{1}, \ldots, \sigma_{n}\right)^{T}, \vec{\omega}^{0}=$ $\left(\omega_{1}, \ldots, \omega_{n}\right)^{T}, \vec{\rho}^{0}=\left(\rho_{1}^{0}, \ldots, \rho_{n}^{0}\right)^{T}, \vec{A}^{0}=\left(A_{1}^{0}, \ldots, A_{n}^{0}\right)^{T}$, and

$$
\begin{aligned}
& S_{1}^{\sigma}=S_{1}^{\omega}=S_{1}^{\rho}=S_{1}^{A}=\left\langle w_{p^{\prime}}(r)\left|\partial_{r}^{2}+2 r^{-1} \partial_{r}\right| B_{p}(r)\right\rangle \\
& S_{2}^{\sigma}=S_{2}^{\omega}=S_{2}^{\rho}=S_{2}^{A}=\left\langle w_{p^{\prime}}(r)\left|r^{-2}\right| B_{p}(r)\right\rangle \\
& S_{3}^{\sigma}=S_{3}^{\omega}=S_{3}^{\rho}=S_{3}^{A}=\left\langle w_{p^{\prime}}(r) \mid B_{p}(r)\right\rangle \\
& S_{4}^{\sigma}=\left\langle w_{p^{\prime}}(r)\left|g_{2} \sigma(r)+g_{3} \sigma(r)^{2}\right| B_{p}(r)\right\rangle .
\end{aligned}
$$

The components of the right hand side vectors are defined as

$$
\begin{gathered}
r_{p^{\prime}}^{(\mathrm{s})}=-g_{\sigma}\left\langle w_{p^{\prime}}(r) \mid \rho_{\mathrm{s}}(r)\right\rangle, \\
r_{p^{\prime}}^{(\mathrm{v})}=g_{\omega}\left\langle w_{p^{\prime}}(r) \mid \rho_{\mathrm{v}}(r)\right\rangle, \\
r_{p^{\prime}}^{(3)}=g_{\rho}\left\langle w_{p^{\prime}}(r) \mid \rho_{3}(r)\right\rangle, \\
r_{p^{\prime}}^{(\mathrm{em})}=e\left\langle w_{p^{\prime}}(r) \mid \rho_{\mathrm{em}}(r)\right\rangle .
\end{gathered}
$$


The nonlinear equation for the $\sigma$-field is solved in an iterative procedure. The nonlinear terms are included in the global stiffness matrix (see $S_{4}^{\sigma}$ in Eq. (A9)). In the iterative solution matrix elements that contain nonlinear terms are calculated using the field $\sigma(r)$ obtained in the previous iteration step.

APPENDIX: REFERENCES

[1] P.G. Reinhard; Rep. Prog. Phys. 52 (1989) 439.

[2] P. Ring; Progr. Part. Nucl. Phys. 37 (1996) 193.

[3] Y.K. Gambhir, P. Ring, and A. Thimet; Ann. Phys. (N.Y.) 511 (1990) 129.

[4] G.Lalazissis, J. König and P. Ring; Phys. Rev. C 55 (1997) 1.

[5] P.G. Reinhard, M.Rufa, J. Maruhn, W. Greiner and J. Friedrich; Z. Phys. A 323 (1986) 13.

[6] D. Vautherin and D.M. Brink; Phys. Rev. C 5 (1972) 626.

[7] J. Decharge and D. Gogny; Phys. Rev. C 21 (1980) 1568.

[8] I. Tanihata et. al, Phys. Rev. Lett. 55 (1985) 2676.

[9] W. Pöschl, D. Vretenar, G.A. Lalazissis and P. Ring; Phys. Rev. Lett. 79 (1997) 3841.

[10] J. Dobaczewski, H. Flocard, and J. Treiner; Nucl. Phys. A422 (1984) 103-139.

[11] K.T.R. Davies, H. Flocard, S. Krieger, M.S. Weiss; Nucl. Phys. A342 (1980) 111-123.

[12] O.A. Marques; CERFACS Report TR/PA/95/31, Toulouse, France.

[13] W. Pöschl, D. Vretenar and P. Ring; Comput. Phys. Commun. 99 (1996) 128-148.

[14] W. Pöschl, D. Vretenar, A. Rummel and P. Ring; Comput. Phys. Commun. 101 (1997) 75-107.

[15] W. Pöschl, D. Vretenar and P. Ring; Comput. Phys. Commun. 103 (1997) 217-251. 
Fig. $3 \mathrm{c}$

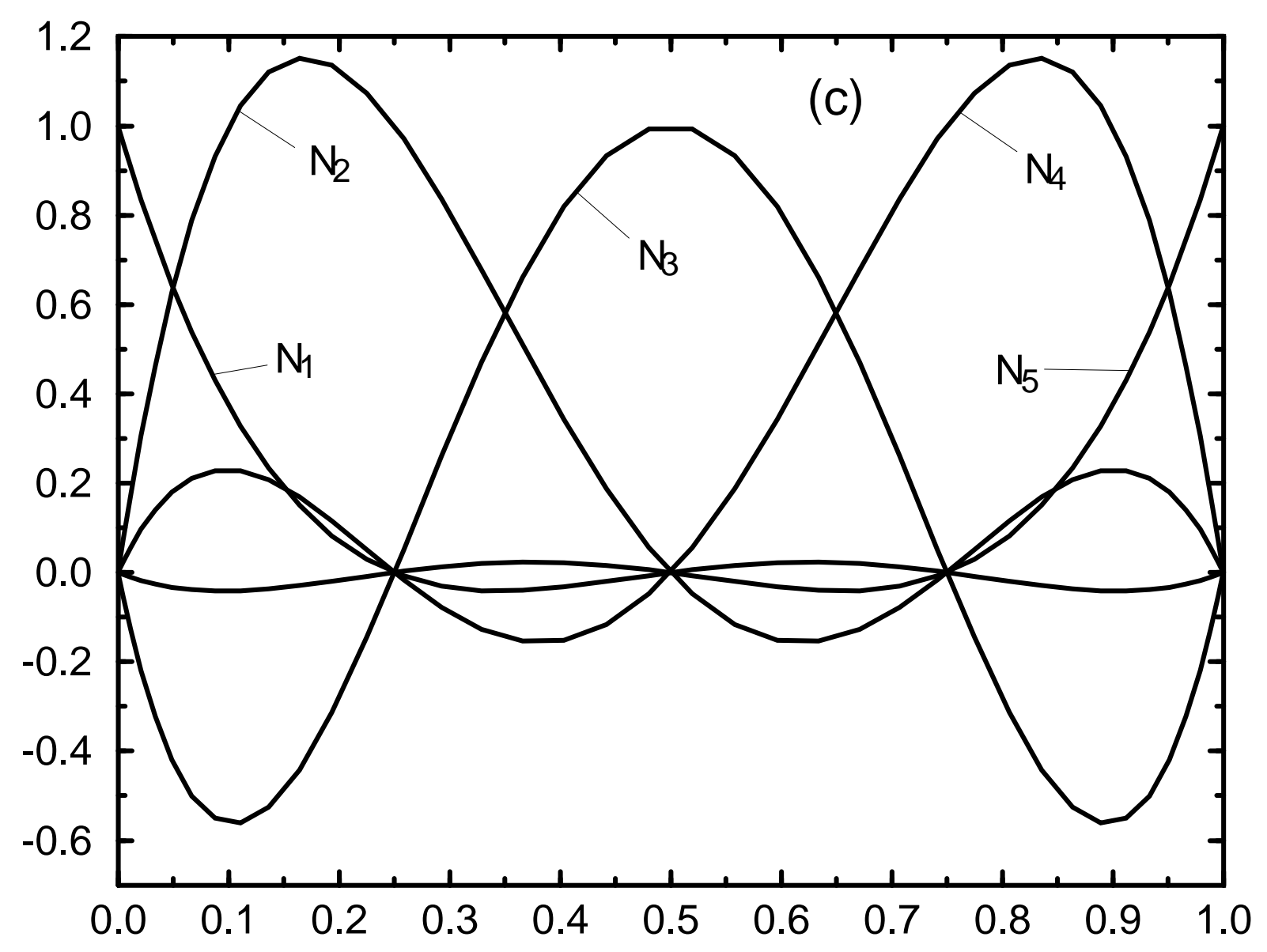


Fig. $3 d$

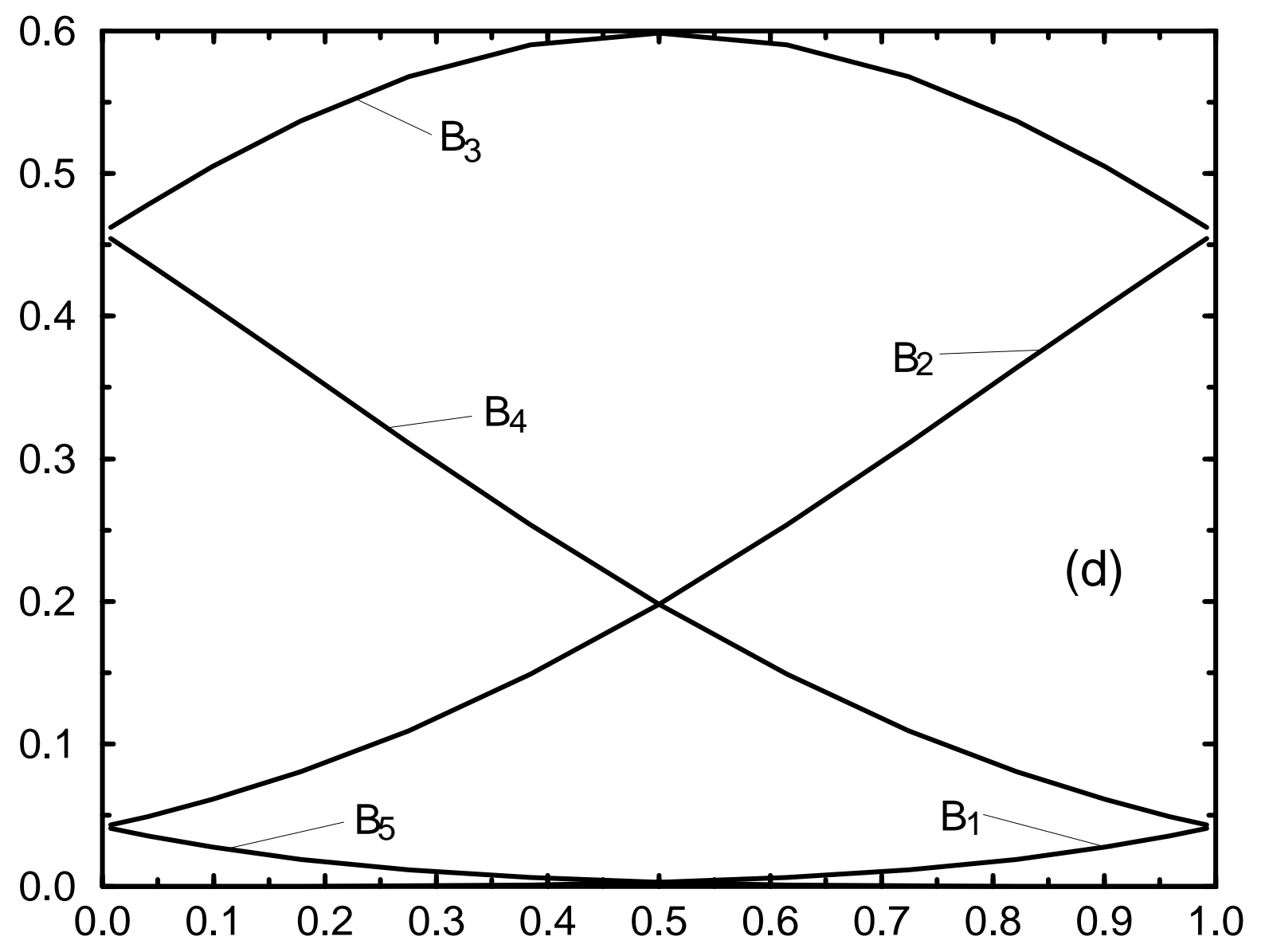


Fig. 3 e

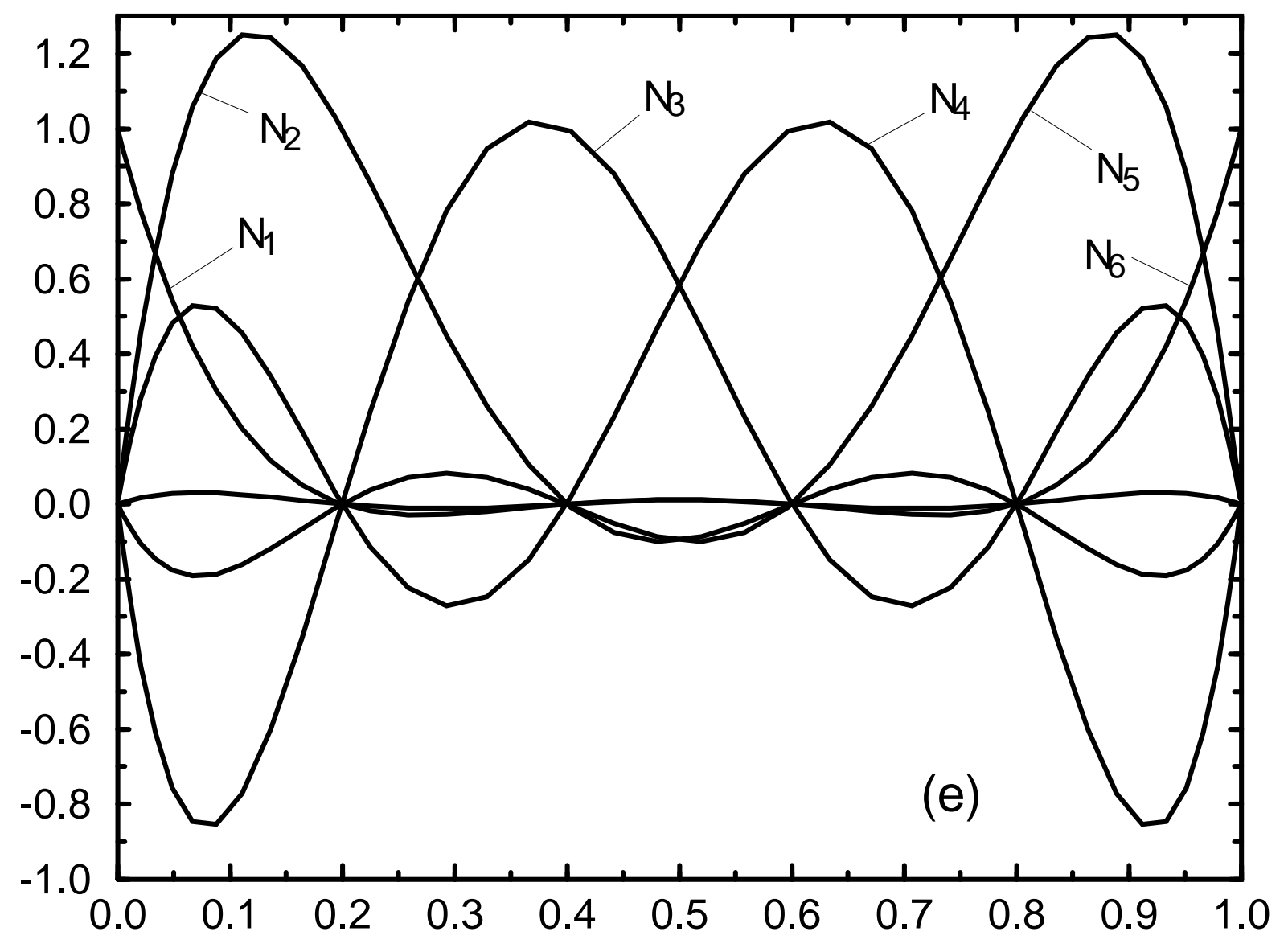


Fig. $3 \mathrm{f}$

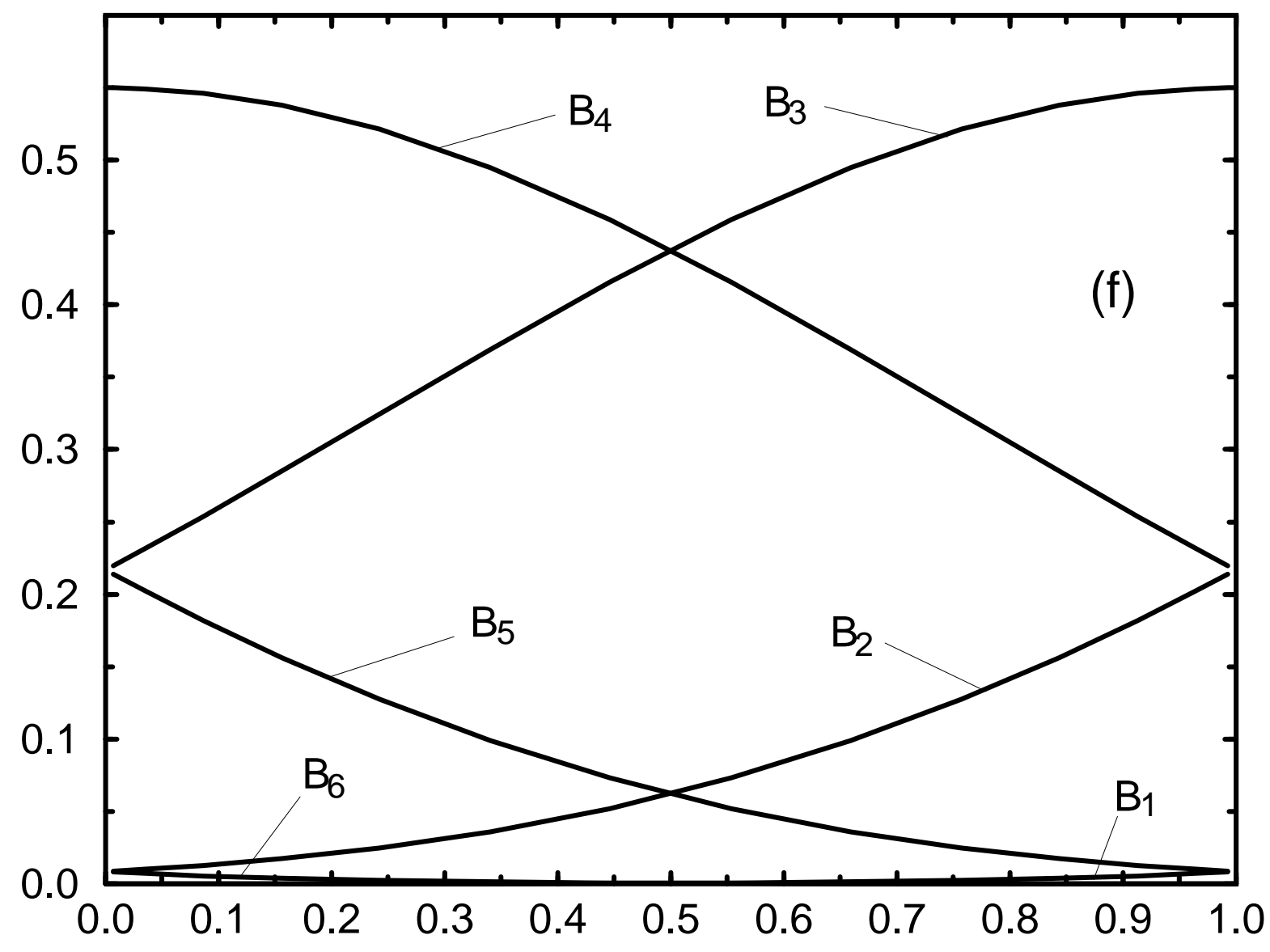

